\title{
Electron-phonon interactions and related physical properties of metals from linear-response theory
}

\author{
S. Y. Savrasov \\ Max-Planck-Institut für Festkörperforschung, Heisenbergstr. 1, 70569 Stuttgart, Germany. \\ D. Y. Savrasov \\ P. N. Lebedev Physical Institute, Leninskii pr.53, 117924 Moscow, Russia
}

(February 1, 2008)

\begin{abstract}
Spectral distribution functions of electron-phonon interaction $\alpha^{2} F(\omega)$ obtained by ab initio linear-response calculations are used to describe various superconducting and transport properties in a number of elemental metals such as $A l, C u, M o, N b, P b, P d, T a$, and $V$. Their lattice dynamics and self-consistently screened electron-phonon coupling are evaluated within local density functional theory and using linear-muffin-tin-orbital basis set. We compare our theoretical $\alpha^{2} F(\omega)$ with those deduced from the tunneling measurements and find a close agreement between them. Temperature dependent electrical and thermal resistivities as well as transport constants $\lambda_{t r}$ also agree well with the experimental data. The values of $\lambda_{t r}$ are close to the electron-phonon coupling parameter $\lambda$. For the later a very good agrement with specific-heat measurements was found without any paramagnon contribution, except in $P d$. We conclude that our method provides the description of electron-phonon interactions in tested materials with the accuracy $10 \%$.
\end{abstract}

\section{INTRODUCTION.}

Electron-phonon interaction (EPI) in metals is a subject of intensive theoretical and experimental investigations. The interest to this problem arises from a variety of physical phenomena such as electrical and thermal resistivities, renormalization of the electronic specific heat (electronic mass enhancement), and, of course, superconductivity, for quantitative understandiag of those a proper description of the EPI is required 1 . Moreover, the reliable estimation of the EPI parameters in particular case of high-temperature superconductivity may be decisive for recognizing the nature of this phenomenon. Unfortunately, even for some transition metals we have controversial experimental and theoretical data related to the estimation of the coupling constant $\lambda$. Analysis is complicated by possible parallel processes of spinfluctuations, for example, in the probleme of renormalization of specific heat and $T_{c}$ or proximity effects in the tunneling data3. To extract the quantities under the interest one has to use some theoretical calculations and models. In this situation fully $a b$ initio calculations of the oneelectron spectra and phonon dispersions based on density functional theoryt (DFT) are most preferable. Applicability of the popular local density approximation $\mathbf{3}$ (LDA) for the functional and treatment of the one-electron band structures as spectra of low-energy electronic excitations were checked many times and there exist a reach theoretical and experimental experience 6 . This allows us to conclude that even having been formally ground-state theory, DFT is a good starting point for investigating the electron-phonon interaction.
Many previous attempts to compute EPI, in particular, for transition metals, have focused on calculat ing merely the electronic contribution to this quantityd, while the phonon frequencies $\omega_{\mathbf{q} \nu}$ and the eigenvectors $\eta_{\mathbf{q} \nu}$ were usually taken from inelastic neutron-scattering data. There, the self-consistent adjustment of the oneelectron potential to the phonon distortion was replaced by either rigid-ion approximation (RIA) or most popular rigid-muffin-tin approximation $\mathrm{S}$ (RMTA). For isotropic metals, having a large density of states at the Fermi energy, the RMTA works well in many cases, since the efficient electronic screening limits the change of the potential in the immediate vicinity of the displaced atom. However, there are known some problems of the RMTA in transition metals. For example, anisotropy of the mass enkancement factor was not reproduced by the RMTA in $N b 10$.

Accurate $\omega_{\mathbf{q} \nu}$ and $\eta_{\mathbf{q} \nu}$ as well as self-consistently screened electron-phonon interaction can be calculated within the total-energy frozen-phonon approach using the supercells 114 . However, there is a serious drawback of this method. Sufficiently large number of phonon wave vectors q must be sampled in the Brillouin zone to get a good estimate of the average coupling strength $\lambda$. A separate frozen-phonon calculation is required for each $\mathbf{q}$ and for each studied mode. For small phonon wave vectors this requires very large supercells. With the crude sampling allowed by the limited size of the supercell, the accuracy of $\mathbf{q}$ integrated quantities like $\lambda$ is usually low.

Another technique which can be employed for calculating the self-consistent change in the potential is the perturbative approach ${ }^{11}$ applicable for any $\mathbf{q}$. Key quantity of this method is an independent-particle polarizability function. After applying the first-order perturbation the- 
ory and expanding the first-order changes in the oneelectron wave functions over the basis of unperturbed Bloch states, the polarizability is expressed via the dowble sum over occupied and unoccupied states. Winter 15 has successfully applied this method to calculate $\lambda$ in Al. Unfortunately, the perturbative approach has several drawbacks. First, the slowly convergent sum over the excited states requires their preliminary calculation by diagonalizing the unperturbed hamiltonian matrix of very large dimension. Second, the self-consistency in this method is done by inverting the dielectric matrix of the crystal which is a relatively time-consuming problem.

To date, the most efficient technique developed to calculate the lattice dynamics is the solid-state generalization 16 of the Sternheimer method 17 . This method is not limited to q's commensurate with the lattice as the frozen-phonon approach and it does not require the knowledge of all unperturbed states as the perturbative approach. The latter advantage can be achieved by constructing a rapidly convergent basis set for representing the first-order corrections to the wave functions other than the basis of unperturbed states. This is important because the first-order corrections as well as the unperturbed wave functions oscillate in the core region. In broad-band semiconductors and insulators this problem can be circumvented by using the pseudopotential method and most calculations of phonon dispersions performed so far use plane-wave basis sets 18 . Unfortunately with decreasing band width the planewave expansion of the pseudo-wave-functions converges more slowly and it becomes less advantageous to use pseudopotentials.

Recently an all-electron formulation of this generalized Sternheimer approach has been given 1921 . The first-order corrections are represented in terms of muffip tin (MT) basis set such as linear-muffin-tin-orbitals22 (LMTO) which greatly facilitates the treatment of localized valence wave functions. For the first time, the method was shown to produce accurate phonon dispersions in transition metals and transition-metal compound\$19 21. In this paper we present details of generalizing this method to compute the wave-vectordependent electron-phonon coppling. (A brief report of this work has appeared already 23 ). We evaluate the spectral distribution functions $\alpha^{2} F(\omega)$ of the EPI from the phonon linewidths $\gamma_{\mathbf{q} \nu}$ according to the approach-developed by Allen 24 in the superconductivity theory 25 . The electron-phonon matrix elements are calculated in the LMTO representation. Due to incompleteness of the basis sets in band-structure calculations the corrections to these matrix elements are shown to exist and explicitly taken into account. The incomplete-basis-set (IBS) corrections appear here in the same manner as in the calculation $\rho f$ the dynamical matrix within the linear-response theory 19 or when calculating the forces within the totalenergy frozen-phonon approach in terms of the LMTO method 26 .

We apply the developed scheme to compute electron-- phonon coupling for a large number of elemental metals. We also present calculations of their phonon dispersion curves. The results of computed transport properties such as temperature-dependent phonon-limited electrical resistivities and thermal conductivities obtained as low-order variational solutions of the Boltzman equation are also given. The method of calculating the transport properties is analogous to that used in the supeconductivity theory and is based27 on calculating the transport spectral functions $\alpha_{t r}^{2} F(\omega)$. All the results presented in this paper are completely ab initio and no adjustable parameters have been used in the calculations.

The rest of the paper is organized as follows. In section II we derive the formulae for calculating the electron-phonon matrix elements and briefly review the method of finding superconducting and transport properties. Section III presents the results of the calculations for phonon dispersions, electron-phonon interactions and related properties for a number of elemental metals such as $A l, C u, M o, N b, P b, P d, T a$, and $V$. Section IV concludes the paper.

\section{METHOD.}

The central problem in calculating the electron-phonon interaction is the evaluation of changes in the electronic Hamiltonian caused by atomic displacements. This generally requires the knowledge of the full low-energy excitation spectrum of the metal: the quasiparticle energies and the phonon frequencies. The calculations of vibrational properties are, in principle, within the scope of the density-functional based methods. Finding the quasiparticle excitation spectra is, on the other hand, much more difficult many-body problem. In the following we always assume that the quasiparticle energies are necessarily approximated by the LDA energy bands.

In the framework of the density-functional linearresponse method the problem of calculating the phonon spectra and the electron-phonon interaction is reduced to finding the first-order variations in the one-electron wave functions, the charge density and the effective potential induced by the presence of a phonon with a given wave vector $\mathbf{q}$. These quantities are connected by the so-called Sternheimer equation, which is the Schrödinger equation to linear order (we use atomic Rydberg units throughout the paper):

$$
\left(-\nabla^{2}+V_{e f f}-\epsilon_{\mathbf{k} j}\right) \frac{\delta^{ \pm} \psi_{\mathbf{k} j}}{\delta R_{\mu}}+\left(\frac{\delta^{ \pm} V_{e f f}}{\delta R_{\mu}}-\frac{\delta^{ \pm} \epsilon_{\mathbf{k} j}}{\delta R_{\mu}}\right) \psi_{\mathbf{k} j}=0,
$$

where $\epsilon_{\mathbf{k} j}, \psi_{\mathbf{k} j}$ are the unperturbed energies and wave functions of vector $\mathbf{k}$ and band $j ; V_{\text {eff }}$ is the effective potential of the DFT; $\delta^{ \pm} \epsilon_{\mathbf{k} j} / \delta R_{\mu}, \delta^{ \pm} \psi_{\mathbf{k} j} / \delta R_{\mu}, \delta^{ \pm} V_{e f f} / \delta R_{\mu}$ are the first-order changes in these quantities induced by the displacements of atoms in the positions $\mathbf{R}+\mathbf{t}(\mathbf{R}$ is the basis vector and $\mathbf{t}$ is the translation vector) moving 
in the direction $\mu$ according to: $\delta \mathbf{t}_{R}=\delta \mathbf{A}_{R} \exp (+i \mathbf{q t})+$ $\delta \mathbf{A}_{R}^{*} \exp (-i \mathbf{q t})$ where $\delta \mathbf{A}_{R}$ is the complex polarization vector. The sign " \pm " in the operator $\delta^{ \pm} / \delta R_{\mu}$ refers to the changes associated with the contributions to $\delta \mathbf{t}_{R}$ which represent two travelling waves of vectors $+\mathbf{q}$ and -q. Note that $\delta^{ \pm} \epsilon_{\mathbf{k} j} / \delta R_{\mu}=\left\langle\mathbf{k} j\left|\delta^{ \pm} V_{e f f} / \delta R_{\mu}\right| \mathbf{k} j\right\rangle$ vanishes unless $\mathbf{q}=0$.

Equation (11) must be solved self-consistently since the induced charge density $\delta^{ \pm} \rho / \delta R_{\mu}$ expressed via $\delta^{ \pm} \psi_{\mathbf{k} j} / \delta R_{\mu}$ screens out the external perturbation $\delta^{ \pm} V_{\text {ext }} / \delta R_{\mu}$. The latter is simply given by the change in the bare Coulomb potential of the nuclei. We thus see that the scheme is the linearized analog of the original Kohn-Sham equations. If Eq.(11) is solved and the value of $\delta^{ \pm} \rho / \delta R_{\mu}$ is available, the phonon frequencies $\omega_{\mathbf{q} \nu}$ and the eigenvectors $\eta_{\mathbf{q} \nu}$ ( $\nu$ numerates phonon branches) are obtained by diagonalizing the adiabatic dynamical matrix of the crystal. The latter is the second-order derivative of the DFT total energy with respect to $\delta R_{\mu}$ and $\delta R_{\mu^{\prime}}^{\prime}$ and is conveniently represented by the HellmannFeynman theorem. In practice, however, there is a problem in using the Hellmann-Feynman expression connected with the fact that the unperturbed wave functions are obtained not as exact solutions of the Kohn-Sham equations but by expanding them into some basis set $\chi_{\alpha}^{\mathbf{k}}$ :

$$
\psi_{\mathbf{k} j}=\sum_{\alpha} \chi_{\alpha}^{\mathbf{k}} A_{\alpha}^{\mathbf{k} j}
$$

Applying the Rayleigh-Ritz variational principle, this leads to finding the unknown coefficients $A_{\alpha}^{\mathbf{k} j}$ from the matrix eigenvalue problem:

$$
\sum_{\alpha}\left\langle\chi_{\beta}^{\mathbf{k}}\left|-\nabla^{2}+V_{e f f}-\epsilon_{\mathbf{k} j}\right| \chi_{\alpha}^{\mathbf{k}}\right\rangle A_{\alpha}^{\mathbf{k} j}=0
$$

The consequence of the variational formulation is that, in general, the Hellmann-Feynman contribution is supplemented with the correction expressed via the derivatives of the basis functions with respect to the displacements, $\delta^{ \pm} \chi_{\alpha}^{\mathbf{k}} / \delta R_{\mu}$, which accounts for the incompleteness of the basis. The correction enters as the matrix element:

$$
\left\langle\sum_{\beta} A_{\beta}^{\mathbf{k} j} \frac{\delta^{ \pm} \chi_{\beta}^{\mathbf{k}}}{\delta R_{\mu}}\left|-\nabla^{2}+V_{e f f}-\epsilon_{\mathbf{k} j}\right| \mathbf{k} j\right\rangle
$$

and is obviously not equal to zero if the states $\psi_{\mathbf{k} j}$ are only variationally accurate. The IBS corrections explicitly disappear for the plane-wave basis set but must be taken into account for the basis of linear muffin-tin orbitals which is used in our work.

To treat properly the changes in the wave functions caused by variation of some external parameters one should check that the basis functions are well adjusted in the whole region of the parameters variations. Within the linear response, this is achieved by representing the first-order perturbations in $\psi_{\mathbf{k} j}$ in the form:

$$
\frac{\delta^{ \pm} \psi_{\mathbf{k} j}}{\delta R_{\mu}}=\sum_{\alpha}\left(\chi_{\alpha}^{\mathbf{k}} \frac{\delta^{ \pm} A_{\alpha}^{\mathbf{k} j}}{\delta R_{\mu}}+\frac{\delta^{ \pm} \chi_{\alpha}^{\mathbf{k}}}{\delta R_{\mu}} A_{\alpha}^{\mathbf{k} j}\right)
$$

where $\delta^{ \pm} A_{\alpha}^{\mathbf{k} j} / \delta R_{\mu}$ are the changes in the expansion coefficients. This is advantageous comparing to the standard perturbation theory, where only the unperturbed states are used as a basis for representing $\delta^{ \pm} \psi_{\mathbf{k} j} / \delta R_{\mu}$ [This is described by only the first contribution in (5)]. The whole expansion (5) must be fastly convergent because the change $\delta^{ \pm} \chi_{\alpha}^{\mathbf{k}} / \delta R_{\mu}$ in the LMTO basis is tailored to the perturbation just like the original LMTO basis $\chi_{\alpha}^{\mathbf{k}}$ is tailored to the one-electron potential.

In order to find the coefficients $\delta^{ \pm} A_{\alpha}^{\mathbf{k} j} / \delta R_{\mu}$ we first construct the functional of the dynamical matrix, minimization of which with respect to $\delta^{ \pm} \psi_{\mathbf{k} j} / \delta R_{\mu}$ leads to the equation (11), and then apply the Rayleigh-Ritz variational principle with the trial functions represented by Eq. This leads to a set of matrix equations for $\delta^{ \pm} A_{\alpha}^{\mathbf{k} j} / \delta R_{\mu}$. The detailed description of this method as well as its applications for calculating phonon dispersions can be found in Refs. 1921.

In this paper we test the produced self-consistent change in the one-electron potential as the potential of electrons interacting with the phonon mode $\omega_{\mathbf{q} \nu}$. Our task is to evaluate the electron-phonon matrix element $g_{\mathbf{k}+\mathbf{q} j^{\prime}, \mathbf{k} j}^{\mathbf{q} \nu}$. The latter is conventionally written in the form:

$$
g_{\mathbf{k}+\mathbf{q} j^{\prime}, \mathbf{k} j}^{\mathbf{q} \nu}=\left\langle\mathbf{k}+\mathbf{q} j^{\prime}\left|\delta^{\mathbf{q} \nu} V_{e f f}\right| \mathbf{k} j\right\rangle
$$

where both states $\psi_{\mathbf{k} j}$ and $\psi_{\mathbf{k}+\mathbf{q} j^{\prime}}$ have the Fermi energy $\epsilon_{F}$ and where the change in the potential is transformed from the Cartesian system to the system associated with the eigenvectors $\eta_{\mathbf{q} \nu}(R \mu)$ of a particular $\mathbf{q} \nu$ - mode:

$$
\delta^{\mathbf{q} \nu} V_{e f f}=\sum_{R, \mu} \frac{\eta_{\mathbf{q} \nu}(R \mu)}{\left(M_{R} \omega_{\mathbf{q} \nu}\right)^{1 / 2}} \times \frac{\delta^{+} V_{e f f}}{\delta R_{\mu}}
$$

\section{$\left(M_{R}\right.$ are the nuclei masses).}

It is not obvious but the expression (6) for $g_{\mathbf{k}+\mathbf{q} j^{\prime}, \mathbf{k} j}^{\mathbf{q} j}$ should be corrected for the incompleteness of the basis functions. This fact immediately follows if we will interpret the matrix element (6) as a splitting of the degenerate band $\epsilon_{\mathbf{k} j}=\epsilon_{\mathbf{k}+\mathbf{q} j^{\prime}}=\epsilon_{F}$ due to the phonon distortion. Consider first a non-degenerate state for which the firstorder correction to the eigenvalue $\epsilon_{\mathbf{k} j}$ is found as a change in the eigenvalue of the matrix problem (3). It is given by

$$
\begin{aligned}
& \delta \epsilon_{\mathbf{k} j}=\left\langle\mathbf{k} j\left|\delta V_{e f f}\right| \mathbf{k} j\right\rangle+ \\
& \left\langle\sum_{\beta} A_{\beta}^{\mathbf{k} j} \delta \chi_{\beta}^{\mathbf{k}}\left|-\nabla^{2}+V_{e f f}-\epsilon_{\mathbf{k} j}\right| \mathbf{k} j\right\rangle+ \\
& \left\langle\mathbf{k} j\left|-\nabla^{2}+V_{e f f}-\epsilon_{\mathbf{k} j}\right| \sum_{\alpha} A_{\alpha}^{\mathbf{k} j} \delta \chi_{\alpha}^{\mathbf{k}}\right\rangle
\end{aligned}
$$

where $\delta \chi_{\alpha}^{\mathbf{k}}$ and $\delta V_{\text {eff }}$ denote the changes in the basis functions and the potential due to some change in the external parameters of the hamiltonian. This formula 
contains both the expression of the standard perturbation theory (first term here) as well as incomplete-basisset corrections [second and third contributions in (8)]. It would be advantageous to use this formula because the eigenvalues of the matrix problem (3) are variationally accurate for the whole range of parameters variation. The same valid if we develop a perturbation theory for the change in the degenerate band $\epsilon_{\mathbf{k} j}=\epsilon_{\mathbf{k}+\mathbf{q} j^{\prime}}$ within (3). This leads to the result:

$$
\begin{aligned}
& g_{\mathbf{k}+\mathbf{q} j^{\prime}, \mathbf{k} j}^{\mathbf{q} \nu}=\left\langle\mathbf{k}+\mathbf{q} j^{\prime}\left|\delta^{\mathbf{q} \nu} V_{e f f}\right| \mathbf{k} j\right\rangle+ \\
& \left\langle\sum_{\alpha} \delta^{\mathbf{q} \nu} \chi_{\alpha}^{\mathbf{k}-\mathbf{q}} A_{\alpha}^{\mathbf{k}+\mathbf{q} j^{\prime}}\left|-\nabla^{2}+V_{e f f}-\epsilon_{\mathbf{k} j}\right| \mathbf{k} j\right\rangle+ \\
& \left\langle\mathbf{k}+\mathbf{q} j^{\prime}\left|-\nabla^{2}+V_{e f f}-\epsilon_{\mathbf{k}+\mathbf{q} j^{\prime}}\right| \sum_{\alpha} \delta^{\mathbf{q} \nu} \chi_{\alpha}^{\mathbf{k}} A_{\alpha}^{\mathbf{k} j}\right\rangle
\end{aligned}
$$

where $\delta^{\mathbf{q} \nu} \chi_{\alpha}^{\mathbf{k}}$ denotes the variation of the MT basis functions due to the phonon distortion of the $\mathbf{q} \nu$-mode and is connected with the variation $\delta^{+} \chi_{\alpha}^{\mathbf{k}} / \delta R_{\mu}$ in the same way as for the induced potential, Eq. (17). The last two contributions in (9) represent the IBS-corrections which are not vanished unless $\psi_{\mathbf{k} j}, \psi_{\mathbf{k}+\mathbf{q} j^{\prime}}$ are the exact solutions.

Formally, the expression (9) can also be obtained by repeating the standard quantum-mechanical derivation of the Fermi "golden rule" for the wave functions represented in terms of the basis set according to (2). The matrix element of the electron-phonon interaction is obtained by considering the scattering rate for transitions from an initial, unperturbed state $\psi_{\mathbf{k} j}(t)$ into a final perturbed state $\tilde{\psi}_{\mathbf{k}^{\prime} j^{\prime}}(t)$ at the time moment $t$ which is given by the overlap integral squared:

$$
\mid\left\langle\mathbf{k} j(t)\left|\widetilde{\left.\mathbf{k}^{\prime} j^{\prime}(t)\right\rangle}\right|^{2}\right.
$$

Since the final state corresponds to the displaced lattice, the best variational estimate for $\tilde{\psi}_{\mathbf{k}^{\prime} j^{\prime}}(t)$ must include the orbitals centered at the new atomic positions and adjusted to the new one-electron potential. To linear-order with respect to the displacements this leads to finding the change in the basis $\chi_{\alpha}^{\mathbf{k}}$ which gives rise to the IBScorrections entered (9).

The expression (9) is thus the linear-response analogy of evaluating the electron-phonon matrix elements via the splitting of the bands in the frozen-phonon supercell method as done in Ref. 14. It is less sensitive to the errors in the wave functions introduced by the variational principle, has a correct long-wavelength behavior and allows one to avoid the inclusion of $d-f$ transitions in $d$-electron systems.

For the electron-phonon spectral distribution functions $\alpha^{2} F(\omega)$ we employ the expression 24 in terms of the phonon linewidths $\gamma_{\mathbf{q} \nu}$

$$
\alpha^{2} F(\omega)=\frac{1}{2 \pi N\left(\epsilon_{F}\right)} \sum_{\mathbf{q} \nu} \frac{\gamma_{\mathbf{q} \nu}}{\omega_{\mathbf{q} \nu}} \delta\left(\omega-\omega_{\mathbf{q} \nu}\right)
$$

where $N\left(\epsilon_{F}\right)$ is the electronic density of states per atom and per spin at the Fermi level. When the energy bands around the Fermi level are linear in the range of phonon energies, the linewidth is given by the Fermi "golden rule" and is written as follows

$$
\gamma_{\mathbf{q} \nu}=2 \pi \omega_{\mathbf{q} \nu} \sum_{\mathbf{k} j j^{\prime}}\left|g_{\mathbf{k}+\mathbf{q} j^{\prime}, \mathbf{k} j}^{\mathbf{q}}\right|^{2} \delta\left(\epsilon_{\mathbf{k} j}-\epsilon_{F}\right) \delta\left(\epsilon_{\mathbf{k}+\mathbf{q} j^{\prime}}-\epsilon_{F}\right) .
$$

The spectral distribution function (11) and its first reciprocal moment $\lambda$ are usually used to describe such important manifestation of the EPI as superconductivity and some normal-state properties. One of such properties is an enhancement of the electronic mass for the electron at the Fermi energy when its velocity $v_{\mathbf{k}}$ is reduced by the factor $1+\lambda_{\mathbf{k}}$ due to the interaction with phonons. The value of $\lambda_{\mathbf{k}}$ is given by the reciprocal moment of the so-called $\mathbf{k}$-dependent electron-phonon spectral function $\alpha_{\mathbf{k}}^{2} F(\omega)$. This renormalization is observed in the de Haasvan Alphen and cyclotron-resonance experiments. As its consequence, the low-temperature electronic specific heat is also renormalized. For the latter effect it is sufficient to know only the Fermi-surface averaged value of $\lambda_{\mathbf{k}}$, i.e. $\lambda$.

Full description of the superconducting state can be obtained by solving the Eliashberg gap equations which relate the energy-gap function and the renormalization parameter for superconducting state to the electronphonon apd the electron-electron interactions in the normal state25. The electron-phonon-coupling function is given by $\alpha^{2} F(\omega)$. The Coulomb interaction is usually represented by some constant $\mu^{*}$. Detailed nature of the effective Coulomb repulsion is not very well known. Fortunately, various definitions for $\mu^{*}$ have only a weak influence on the solution of the gap equations and its values can, e.g., be found by adjusting the calculated transition temperatures to the experimental ones.

Electron-phonon scattering has a dominant contribution to the electrical resistivity for reasonably pure metals except very low-temperature region where the impurity and electron-electron scattering are important. The influence of the EPI on the transport properties are described in terms of the transport spectral functioned $\alpha_{t r}^{2} F(\omega)=\alpha_{\text {out }}^{2} F(\omega)-\alpha_{i n}^{2} F(\omega)$ where:

$$
\begin{aligned}
& \alpha_{\text {out (in) }}^{2} F(\omega)= \\
& \frac{1}{N\left(\epsilon_{F}\right)\left\langle v_{x}^{2}\right\rangle} \sum_{\nu} \sum_{\mathbf{k} j \mathbf{k}^{\prime} j^{\prime}}\left|g_{\mathbf{k}^{\prime} j^{\prime}, \mathbf{k} j}^{\mathbf{k}^{\prime}-\mathbf{k}}\right|^{2} v_{x}(\mathbf{k}) v_{x}\left(\mathbf{k}^{\left({ }^{\prime}\right)}\right) \times \\
& \delta\left(\epsilon_{\mathbf{k} j}-\epsilon_{F}\right) \delta\left(\epsilon_{\mathbf{k}^{\prime} j^{\prime}}-\epsilon_{F}\right) \delta\left(\omega-\omega_{\mathbf{k}^{\prime}-\mathbf{k} \nu}\right)
\end{aligned}
$$

Here $\left\langle v_{x}^{2}\right\rangle$ is the average square of the $x$ component of the Fermi velocity. In the lowest-order variational approximation (LOVA) for the solution of the Boltzman equation the expressions for electrical and thermal resistivities are

$$
\rho(T)=\frac{\pi \Omega_{c e l l} k_{B} T}{N\left(\epsilon_{F}\right)\left\langle v_{x}^{2}\right\rangle} \int_{0}^{\infty} \frac{d \omega}{\omega} \frac{x^{2}}{\sinh ^{2} x} \alpha_{t r}^{2} F(\omega),
$$




$$
\begin{gathered}
w(T)=\frac{6 \Omega_{\text {cell }}}{\pi k_{B} N\left(\epsilon_{F}\right)\left\langle v_{x}^{2}\right\rangle} \int_{0}^{\infty} \frac{d \omega}{\omega} \frac{x^{2}}{\sinh ^{2} x} \times \\
{\left[\alpha_{t r}^{2} F(\omega)+\frac{4 x^{2}}{\pi^{2}} \alpha_{\text {out }}^{2} F(\omega)+\frac{2 x^{2}}{\pi^{2}} \alpha_{\text {in }}^{2} F(\omega)\right]}
\end{gathered}
$$

with $x=\omega / 2 k_{B} T$. The LOVA results (13), (14) give the upper bound to the resistivities and allow us to test the calculated $\alpha_{t r}^{2} F(\omega)$.

\section{RESULTS.}

\section{a. Technicalities.}

Our calculations of phonon dispersions and electronphonon interactions for the elemental metals such as fcc$\mathrm{Al}, \mathrm{Cu}, \mathrm{Pb}, \mathrm{Pd}$, and bcc- $\mathrm{Mo}, \mathrm{Nb}, \mathrm{Ta}, \mathrm{V}$ are performed in the framework of the linear-response LMTO method21. The details of the calculations are the following: We find the dynamical matrix and the phonon linewidths for these materials as a function of wave vector for a set of irreducible q-points at the $(8,8,8)$-reciprocal lattice grid [29 points per $1 / 48$ th part of the Brillouin zone (BZ)]. The $(I, J, K)$ reciprocal lattice (or Monkhorst-Pack 28 ) grid is defined in a usual manner: $\mathbf{q}_{i j k}=\frac{i}{I} \mathbf{G}_{1}+\frac{j}{J} \mathbf{G}_{2}+$ $\frac{k}{K} \mathbf{G}_{3}$, where $\mathbf{G}_{1}, \mathbf{G}_{2}, \mathbf{G}_{3}$ are the primitive translations in the reciprocal space. The self-consistent calculations performed for every wave vector involve the following parameters: We use $3 \kappa-s p d-$ LMTO basis set (27 orbitals) with the one-center expansions performed inside the MT-spheres up to $l_{\max }=6$. In the interstitial region the basis functions are expanded in plane waves up to the cutoff approximately corresponding to 70,140 , and 200 plane waves per $s, p$, and $d$-orbitals, respectively. All semicore states lying higher than $-4 R y$ are treated as valence states in separate energy windows. The induced charge densities and the screened potentials are represented inside the MT-spheres by spherical harmonics up to $l_{\max }=6$ and in the interstitial region by plane waves with the cutoff corresponding to the $(16,16,16)$ fastFourier-transform grid in the unit-cell of direct space.

The $\mathbf{k}$-space integration needed for constructing the induced charge density and the dynamical matrix is performed over the $(16,16,16)$ grid $(145$ points per $1 / 48$ th part of the BZ) which is twice denser than the grid of the phonon wave vectors $\mathbf{q}$. We use the improved tetrahedron method of Ref. 29. However, the integration weights for the $\mathbf{k}$-points at this $(16,16,16)$ grid have been found to take precisely into account the effects arising from the Fermi surface and the energy bands. This is done with help of the energies $\epsilon_{\mathbf{k} j}$ generated by the original full-potential LMTO method at the $(32,32,32)$ grid $(897$ points per $1 / 48 \mathrm{BZ}$ ). The procedure is in details explained in Ref. 21 and allows us to obtain more convergent results with respect to the number of $\mathbf{k}$ points.

The $\mathbf{k}$-space integration for the phonon linewidths $\gamma_{\mathbf{q} \nu}$ is very slowly convergent because it involves two $\delta$ functions according to Eq. (12). It is performed with help of the $(32,32,32)$ grid in the BZ by means of the tetrahedron method of Ref. 30. The largest numerical error of $\alpha^{2} F(\omega)$ comes from the integration over $\mathbf{q}$ in the expression (11). Its magnitude, we estimated by performing the integration over merely the band-structure factor [which is $\gamma_{\mathbf{q} \nu}$ approximated by $\left.\sum_{\mathbf{k} j j^{\prime}} \delta\left(\epsilon_{\mathbf{k} j}-\epsilon_{F}\right) \delta\left(\epsilon_{\mathbf{k}+\mathbf{q} j^{\prime}}-\epsilon_{F}\right)\right]$ using respectively $(8,8,8)$ and $(32,32,32)$ grids and found to be not larger than $7 \%$ in all cases.

A few words should be said about the lattice parameters used in the calculations. It is known that the equilibrium cell volume $V$ found theoretically by the corresponding LDA-based total-energy calculation is frequently obtained slightly lower than the experimental volume $V_{0}$. This usually leads to the calculated at $V_{0}$ phonon frequencies which are softer comparing to the experimental ones. Often, a better agreement with the experiment can be obtained by performing the linearresponse calculations at the theoretical volume. This is, in principle, a justified procedure from theoretical point of view. Unfortunately, the prescription does not work when calculating the phonon linewidths and $\alpha_{t r}^{2} F(\omega)$ : the results of calculated electrical and thermal resistivities agree less well with the experiment. The reason for this discrepancy is connected with the sensitivity of these quantities to the shape of the Fermi surface. It turns out that the use of the Fermi surfaces calculated at the experimental lattice constants considerably improves the results. We can thus use theoretical volumes in the linear-response calculations of phonon dispersions and the electron-phonon matrix elements. To find the phonon linewidths and $\alpha_{t r}^{2} F(\omega)$ we can use, on the other hand, the energy bands entered (12) which are generated at the experimental lattice constants. We understand that it is not well justified procedure to use different lattice parameters in one calculation, but it somewhat helps to minimize the errors connected with the LDA by simple means. The actual volume ratios $V / V_{0}$ used in our calculations to find the changes in the one-electron potentials are listed in Table I.

Another comment concerns the choice of the exchangecorrelation potential. The general strategy employed by us is to use the exchange-correlation formula which gives the best prediction of the cell volumes. The BarthHedin-like formula after Ref. 31 is employed for all the metals except $C u$ and $V$. For the $3 d$-metals we have found that this formula gives the theoretical volumes which are too small $\left(V / V_{0} \sim 0.9\right)$. For $C u$ and $V$ the Ceperley-Alder form 32 of the exchange-correlation potential parametrized after Ref. 33 is used which gives the ratios $V / V_{0}$ being much closer to unity (see Table I).

Finally note that in the previous publications $192,22,23$ we have used a different method for treating the fullpotential terms in the calculation which was based on the atomic cells and the one-center spherical-harmonic expansions26. This method is not directly applicable 
to calculate phonon dispersions for materials with open structures such, e.g., the diamond structure and requires the replacement of empty sites of the lattice by empty spheres. This complicates the evaluation of the dynamical matrix. In recent publication 21 we have employed another approach based on the plane-wave expansions for the LMTOs in the interstitial region and have applied the method to calculate the phonon spectra in $S i$ and $N b C$. While the materials considered in this work have close-packed bcc or fcc structures we also apply this new method which is more general for practical use. Some the results for $A l, N b$, and $M o$ previously published 19,2023 do not noticeably differ from those presented below in this paper.

\section{b. Lattice-dynamical properties.}

In Table I we report the values of the calculated phonon frequencies at the high-symmetry points $X$ and $L$ for the fcc metals $A l, C u, P b$ and $P d$ as well as at the points $H$ and $N$ for the bcc metals $M o, N b, T a$, and $V$. For the comparison, the experimental frequencies 34 are also listed in Table I along with the theoretical-to-experimental volume ratios which have been used in the calculations.

Our results for the phonon dispersions along several symmetry directions together with the corresponding densities of states for these materials are displayed in Fig.1(a)-(h). The theoretical lines result from the interpolation between the calculated frequencies which are denoted by circles. Many neutron-diffraction measurements are available 34 for nearly all the metals considered here and these data are also shown in Fig.1 by triangles. The only exception is $V$ for which the dispersion relations cannot be studied with neutrons since $V$ is an almost totally incoherent neutron scatterer. While some $X$-ray diffraction measurements exist in the literature 34 their accuracy seems to be less satisfactory than corresponding neutron-scattering data for other materials and we do not show the experimental points for $V$.

From Fig.1 we see that the agreement between theory and experiment is good. Most of the calculated frequencies agree within a few percent with those measured. This also follows from the numerical values listed in Table I. In particular, for $A l$ [Fig.1(a)] a very good agreement is found in all the directions. As we have mentioned already, this calculation is performed at the theoretical volume $\left(V / V_{0}=0.955\right)$. We have also checked the set-up with the experimental volume and found a considerable softening (about 20\%) of the transverse modes. This illustrates the importance of performing lattice-dynamical calculations at the theoretical volumes.

The most important consequence of our calculation for lead [Fig.1(b)] is that the pronounced dip of both the longitudinal and transverse branches near the $X$-point is well reproduced. We have also found a slight overestimate of the transverse phonon frequencies near this zone boundary which can be attributed neither to the dis- crepancy in the cell volume $\left(V / V_{0}=1.002\right)$ nor to the neglection of the semicore states. (We have in fact included both $5 d$, and $6 d$-states in the main valence panel). This kind of disagreement has also been recently reported in Ref. 35 using the linear-response pseudopotential technique. It is possibly connected with the use of the local density approximation or the lack of spin-orbit coupling effects in our calculation.

The most interesting cases are $V, N b$, and $T a$ [Fig.1(c),(d),(e), respectively]. The materials belong to Group $\mathrm{V}$ of the periodic table and all of them show anomalious behavior of the phonon dispersion curves. The presence of anomalies is, first of all, connected with the well-known dip of the longitudinal mode in the $(00 \xi)$ direction. The dip is correctly reproduced by our calculation. Another important features of our calculation are (i) the softening of the transverse mode along the $(00 \xi)$ direction at long wavelengths which is rather sharp in both $\mathrm{V}, \mathrm{Nb}$ and is weaker in $\mathrm{Ta}$ as well as (ii) the crossover of two transverse branches in the $(\xi \xi 0)$ direction. The latter is in fact predicted for $T a$ because the measured dispersions in this direction are absent for the $T_{1}$-branch except the zone-boundary point $N$.

The theoretical phonon dispersions for $M o$ also agree well with the experiment. The consequences of our calculations here are the reproduced softening near the $H$ point and the absence of the large dip along the $(\xi \xi \xi)$ direction near $\xi \sim 0.7$. The dip is presented in the dispersion curves of nearly all bcc metals except of $M o$ and $\mathrm{Cr}$, and is certainly the feature of the behavior of the crystalline structure factor which enters the dynamical matrix as a sum over lattice vectors with the phase shift $\exp (i \mathbf{q t})$. Its absence indicates a considerable wavevector dependence of the electron-phonon matrix elements which seems to be well reproduced by our method.

Finally we compare the results of our calculations for $C u$ and $P d$ which are presented in Fig.1(f) and Fig.1(g). The dispersion relations for these materials do not show the anomalies and are smooth. A slight overestimation of the theoretical phonon frequencies is found for both these metals. The overestimation can likely be corrected by the use of the cell volumes in the calculation which would be slightly larger than those found within the LDA. However, such a procedure is not justified theoretically. We think that employing the gradient-corrected density functional 36 known to predict much better the equilibrium lattice parameter will allow us to improve these results.

\section{c. Superconducting properties.}

We now discuss applications of our ab initio linearresponse method to calculate the electron-phonon coupling and superconducting-state properties. First, we present our results for the spectral distribution functions and $\lambda$. Second, we describe our applications to solving the Eliashberg gap equations. 
Calculated $\alpha^{2} F(\omega)$ for $A l$ is shown in Fig.1(a) (full line). The positions of the maxima here are conditioned by the form of the phonon density of states with the low-frequency phonon peak suppressed by the coupling function $\alpha^{2}(\omega)$ (dashed line). The broad phonon spectrum in $A l$ is extended up to the maximal frequency $\omega_{\max } \approx 470 K$. The theoretical $\alpha^{2} F(\omega)$ in Fig.1(a) is com pared with the results of the tunneling measurements 3 (squares). We find a rather good agreement between the two curves. In fact, our $\alpha^{2} F(\omega)$ is also found to be practically identical to the empirical pseudopotential result of Ref. 37 based on the rigid-ion approximation. The latter is known to work well in simple metals. General agreement is found between our and the $a b$ initio frozenphonon results of Dacorogna et. al 12 for the dispersion of the phonon linewidths along the high-symmetry directions. The only exception is that, in the $(00 \xi)$ direction, our longitudinal branch of $\gamma_{\mathbf{q} \nu}$ exceeds theirs by a factor of 2 . This is presumably connected with replacing the $\delta$ functions in (12) by Gaussians used in Ref. 12. However, the relative weight of our high $\gamma$ values in the integrated quantities, such as $\alpha^{2} F(\omega)$ and $\lambda$, is found to be very small. Our value of $\lambda$ is 0.44 which is very close to the value of $\lambda_{\text {tun }}=0.42$ extracted from the tunnelling measurements 3 . The frozen-phonon 12 and linear-response calculations of Winter 15 gave, respectively, $\lambda=0.45$ and 0.38 . The value of $\lambda_{s-h}$ extracted from the electronic specific-heat coefficient $\gamma$ and our calculated density of states $N\left(\epsilon_{F}\right)$ using the relation

$$
1+\lambda_{s-h}=\frac{3 \gamma}{2 \pi^{2} k_{B} N\left(\epsilon_{F}\right)},
$$

is 0.43 (see Table II). In order to check previous conclusions 15.43 about the inapplicability of the RMTA for $s p$ metals, we also performed such a calculation and indeed found $\lambda_{R M T A}=0.14$.

Lead is a well-studied classical example of strongcoupled superconductor with $T_{c}=7.19 \mathrm{~K}$ and its tunnelling spectra have been studied a long time ago 38 . Obtained $\alpha^{2} F(\omega)$ using our linear-response method is presented in Fig.2(b) where it is compared with the results of the measurements 38 . The two curves are similar. Our calculated $\lambda=1.68$ is found to be $8 \%$ larger than the tunnelling value 1.55 and only $2 \%$ larger than the value 1.64 extracted from specific-heat data (see Table II). This disagreement is well within the accuracy of our calculation.

We now report our results for $V, N b$ and $T a$ which are the best-studied elemental superconductors because of their relatively high- $T_{c}$ values. Especially, for $\mathrm{Nb}$ which has the highest $T_{c}=9.25 \mathrm{~K}$ among the elemental metals, there exist many experimental investigations of the tunneling spectra 3942 and theoretical RMTAbased calculations 43 . Unfortunately, some of the results which have been reported in the literature are controversial. First, the RMTA calculations give the values of $\lambda$ varying from 1.12 to 1.86 . Second, the tunneling estimates of the coupling constant for $\mathrm{Nb}$ and $V$ are dif- ficult because of the oxidation of the surface layers. Having lower transition temperature, such oxides act on the tunneling spectra due to the proximity effect. The experimental $\lambda$ in $N b$ varied in the past from the values 39 $0.58-0.68$ with negative or anomaliously small $\mu^{*}$ to the value 40.9 . At present, a satisfactory explanation of the anomalious behavior of the thermally oxidized tunnelling junctipns in $N b$ appears to be possible which gives the values 41,42 of $\lambda_{\text {tun }}=0.92 \div 1.22$.

In Fig.2(c),(d),(e) we present our calculated $\alpha^{2} F(\omega)$ (full lines) for $V, N b$ and $T a$, respectively. They all have rather broad spectra extended up to $\omega_{\max } \approx$ $370 K, 310 K$, and $240 K$. The coupling function $\alpha^{2}(\omega)$ (dashed line) in these metals only slightly deviates from constant in major part of the frequencies. The approximation $\alpha^{2}=$ const. works very well in $N b$ and $T a$. This qualitative result implies that the electron-phonon coupling can be factorized into electronic and phonondependent factors 47 .

In Fig. 2 the calculated spectral functions are compared with the results of the tunneling measurements (squares).

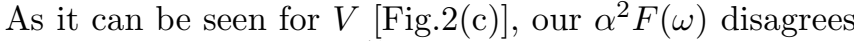
with the measured ond 49 because of the appearance of the upper phonon peak not presented in the experiment. Even though the theoretical $\alpha^{2} F(\omega)$ should be broadened because the $\delta$ function in Eq. (11) ought to be a Lorentzian of half-width $\gamma_{\mathbf{q} \nu}$, the electron-phonon coupling estimated by us $(\lambda=1.19)$ is $40-50 \%$ stronger than the obtained $\lambda_{\text {tun }}=0.82$. The same situation is found for $N b$. Our calculation here [Fig.2(d)] also does not show the suppression of the longitudinal peak. The latter is absent in nearly all the experiments for this metall1]. [A typical measured spectrum 3 is shown in Fig.2(d) by squares]. As a result, the calculated $\lambda=1.26$ is $20 \%$ higher than $\lambda_{\text {tun }}=1.043$. The discrepancy found by us has already been reported in the past RMTA-based calculations 44.45. To check the consistency of our results with the earlier ones, we have performed our own RMTA calculations and obtained complete agreement between them. We thus conclude that the full inclusion of screening does not resolve the problem of the suppressed longitudinal peak.

Unfortunately, our comparison with the experiment is complicated by the proximity effect and the extraction of the tunneling densities of states depends on the way how the measured data are processed. For example, in $N b$ the value of $\lambda_{t u n}=1.22$ deduced from the tunneling experiments (which is only $3 \%$ lower than that found by us) has been reported in the literature 12 . The obtained $\alpha^{2} F(\omega)$ [denoted in Fig.2(d) by triangles] is found much closer to our calculation.

A better understanding of the present situation can be achieved by comparing the theoretical and the experimental 50, tunneling spectra for $T a$ since its superconducting properties are close to those of $V$ and $\mathrm{Nb}$ but this metal is much less reactive with oxygen. Such a comparison is given in Fig.2(e). We find rather good agreement between the both curves. In particular, the 
upper phonon peak is not suppressed in the measured $\alpha^{2} F(\omega)$ and its amplitude is comparable with that calculated by us. As a result, the theoretical $\lambda=0.86$ agrees within $10 \%$ with the $\lambda_{\text {tun }}=0.78$.

From view of the data on $T a$ we cannot consider the discrepancy found for $V$ and $N b$ as a drawback of either our linear-response method or the use of the local density approximation. Partially this conclusion is also verified by alternative estimates of the coupling constant based on the specific-heat data and the de Haas-van Alphen (dHvA) experiments, but it should be noted that both cyclotron masses and the specific-heat coefficient are also enhanced by the electron-electron interactions. Evaluation of the average coupling from the specific-heat measurement.54 yields an enhancement of 1.00 for $V$ and 1.17 for $N b$ (see Table II). If one uses a more recent value 55 of $\gamma$ for $V$ rather than listed in Ref. 54 one obtains the enhancement $\lambda_{s-h}=1.17$ which is nearly coincides in this metal with our $\lambda=1.19$. Comparison between the IDA band masses with those measured by dHvA effect 51 yields the enhancement of 1.33 for $N b$, which is close to the value 1.26 found in our calculation. Another important result is that the measured variation of the mass enhancement for the various cyclotron orbits in $\mathrm{Nb}$ agrees also well with our calculation. Namely, we have found a decomposition of $\lambda$ by the Fermi-surface sheets: (i) octahedron, (ii) jungle gym and (iii) ellipsoids, and have obtained the contributions $\lambda^{\mathrm{i}}=1.44, \lambda^{\mathrm{ii}}=1.37$, and $\lambda^{\mathrm{iii}}=1.08$. They can be compared with the measured $\lambda_{d H v A}^{\mathrm{i}}=1.71, \lambda_{d H v A}^{\mathrm{ii}}=1.43$, and $\lambda_{d H v A}^{\mathrm{iii}}=1.10$. (Note that the calculated within the RMTA anisotropy of the mass enhancement strongly disagrees with these data51.) Moreover, we have estimated the transport constants $\lambda_{t r}$ for these metals both from the calculated and measured resistivity data. The values of $\lambda_{t r}$ are usually believed to be close to the superconducting ones. (A complete report of our calculated transport properties will be given in the following subsection). For $V$, the value of $\lambda_{t r}$ found by us is 1.15 and, for $N b, \lambda_{t r}=1.17$. The calculated electrical and thermal resistivities are also close to those measured. It therefore seems that our electronphonon coupling is accurate (within the computational accuracy of order 10\%) while the effect of the electronelectron interactions is small in these metals.

As the next two examples, we report the results of our applications for $\mathrm{Mo}$ and $\mathrm{Cu}$. There are no tunneling data for these materials because of their low $T_{c}$ and the weakness of phonon effects. The calculated spectral functions are presented in Fig.2(f),(g). Both curves qualitatively agree with the corresponding phonon state densities shown for these metals in Fig.1(f),(g) but a considerable frequency dependence of the electron-phonon prefactor $\alpha^{2}(\omega)$ (indicated by dashed lines) is also predicted. For $M o$ our linear-response calculations are found to be close to our RMTA calculations and to earlier ones 52 . The estimated average coupling here is 0.42 which can be compared with the value 0.45 deduced from the specificheat measurements (see Table II). The calculated value of $\lambda$ for $C u$ is 0.14 . The specific-heat estimate here is less reliable possibly because of the smallness of $\lambda$ and errors due to the experimental uncertainty in the value of $\gamma$. Quite likely, however, that there are some errors in the DFT value of the density of states connected with the many-body effects since copper valence shell $3 d^{10}$ is close to the strongly correlated $3 d^{9}$ configuration. It is known that within DFT the position of $d$-band is higher than experimentally observed. The band which crosses the Fermi level is essentially $s$-band but the effect of hybridization with the $d$-band should lead to lowering the Fermi velocities. The latter effect is stronger if the $d$-band is closer to the Fermi energy. To obtain $1+\lambda_{s-h} \sim 1.1$, one has to reduce our calculated $N\left(\epsilon_{F}\right)$ by approximately $20 \%$ which is a reasonable estimate for the expected influence of the Coulomb correlations. Concerning other estimates of $\lambda$ based on the transport properties, our calculated $\lambda_{t r}=0.13$ while this value extracted from the measured resistivity data is 0.12 (see following subsection). Both values are in agreement with our superconducting $\lambda$.

As the last example, we consider $P d$ and discuss paramagnon effects. The superconductivity in $P d$ is absent because of the large spin fluctuations. There was also a discussion in the literature 53 on the paramagnon contributions to the mass enhancement in $N b$ and $V$. The occurrence of paramagnons is connected with the fluctuations of the electron spins. Paramagnons usually counteract superconductivity since the latter has its origin in the formation of pairs with the opposite spins. To extract the paramagnon contribution, we can use our calculated values of $\lambda$ together with the specific-heat 54 estimates $\lambda_{s-h}$ after formula (16). The necessary data are listed in Table II. Comparing these results does not leave any place for $\lambda_{\text {spin }}=\lambda_{s-h}-\lambda$ in all the materials except in $P d$ which is a typical example for paramagnon effects. Here $\lambda_{s-h}=0.69$ and with the use of our calculated $\alpha^{2} F(\omega)$ [Fig.2(h)], the average electron-phonon coupling is found to be equal to 0.35 . This results in our value of $\lambda_{\text {spin }}=0.34$ for $P d$ which is close to its earlier estimate 0.31 based on the RMTA calculation 46 .

After comparing the calculated and experimental spectral functions, we present the results of our applications to solving the Eliashberg gap equation with our knowledge of $\alpha^{2} F(\omega)$. Having fixed the Coulomb pseudopotential $\mu^{*}$, the superconducting state is now completely described by the strong-coupling theory of superconductivity25 According to the Allen-Dynes 48 modified McMillan 47 formula:

$$
T_{c}^{M c M}=\frac{\omega_{\log }}{1.2} \exp \left(-\frac{1.04(1+\lambda)}{\lambda-\mu^{*}(1+0.62 \lambda)}\right)
$$

the effect of the first reciprocal moment $\lambda$ of $\alpha^{2} F(\omega)$ on $T_{c}$ is most important. Unfortunately, the estimation of the coupling constant from $T_{c}$ is difficult because of the unknown value of $\mu^{*}$. We use standard Matsubara technique to solve numerically the Eliashberg equation for $T_{c}$ 
and have found $\mu^{*}$ which gives the experimental value of $T_{c}$. The cutoff parameters $\omega_{c u t}$ were taken to be equal to ten phonon-boundary frequencies $\omega_{\max }$. To treat the Coulomb pseudopotential in terms of the expression (17) when solving the Eliashberg equation we have rescaled actually used parameters $\mu^{*}\left(\omega_{\text {cut }}\right)$ to $\mu^{*}=\mu^{*}\left(\omega_{\text {log }}\right)$ according to

$$
\frac{1}{\mu^{*}}=\frac{1}{\mu^{*}\left(\omega_{c u t}\right)}+\ln \frac{\omega_{c u t}}{\omega_{l o g}} .
$$

Table III reports the obtained $\mu^{*}$ values. The main conclusion here is that the calculated $\mu^{*}$ varies between 0.11 and 0.17 which is close to the conventional value usually taken $\sim 0.13$. The noticeable exceptions are only $N b$ and, especially, $V$ for which too large $\mu^{*}$ have been found. Of course, this overestimation occurs over the conventional quantity 0.13 while the detailed theoretical data on the Coulomb pseudopotential are unknown. We think that the obtained quantities are still below the upper limit for the allowed $\mu^{*}$ values.

Also listed in Table III are the values of $T_{c}^{M c M}$ evaluated after (17) with our calculated $\omega_{l o g}, \lambda$ and $\mu^{*}$. As it can be seen, the exact solution of the Eliashberg equation gives $T_{c}$ (chosen to be the experimental value) which slightly deviates from that estimated after the McMillan expression. The accuracy of the later is averagely about $15 \%$.

To conclude that our spectral functions provide a proper description of superconductivity we have found the energy-gap parameters $\Delta_{0}$. These results are shown in the last two rows of Table III. The available tunneling data for $A l$ give $\Delta_{0}$ coinciding with the theoretical value. Some overestimation of the coupling constant in comparing to the experimental one has taken place in $\mathrm{Pb}$, while $\Delta_{0}$ agrees very closely. Let us turn out to the important case of transition metals $V, N b$ and $T a$. As we have discussed already, the main difficulties in the tunneling studies in $V$ and $N b$ are connected with the oxidation of the surface layers and the tunneling estimations of the coupling constant in $\mathrm{Nb}$ vary considerably in the past. In contrast to it, the measured superconducting gap in $\mathrm{Nb}$ has approximately the same yalues in all the experiments and is equal to $1.56 \mathrm{meV}$. This value perfectly agrees with that found by us which is equal to $1.53 \mathrm{meV}$. We have also found a good agreement in the energy gap for $V$ which is within $4 \%$ of the experimental one. The discrepancy in the energy gap for $T a$ is again $1-2 \%$. Because of the low transition temperature there is no tunneling data for $M o$ and we only give the theoretical value of $\Delta_{0}$. We thus see that despite of the discrepancy in evaluating the coupling constants, an extremely good agreement $(1-2 \%)$ is obtained for the predicted gap data. Such a coincidence is readily understood because the ratio $2 \Delta_{0} / T_{c}$ is slowly varying for different superconductors. (It is 3.52 within the BCS theory.) Fixing the $T_{c}$ to its experimental value makes the value of $\Delta_{0}$ insensitive to the errors in $\alpha^{2} F(\omega)$ and $\lambda$.
To summarize, we have found that our results for the spectral functions and, in particular, for the coupling constants are realistic for the correct description of the superconducting properties. Especially, an excellent agreement has been found between our calculated $\lambda$ and the values extracted from specific-heat measurements. The values of $\lambda$ deduced from the available tunnelling experiments also agree within $10 \%$ with our calculations in all the materials except $N b$ and, especially, $V$. However, taking into account the past tendency to correct the tunneling spectra for $N b$ as well as our calculations for $T a$, we do not consider these discrepancies as essential.

\section{d. Transport properties.}

We now report the results of our applications for calculating the electron-phonon contribution to the electrical and thermal resistivities (conductivities). This field retains very important and interesting, first, because the easily measured transport properties and, especially, the electrical resistivities, provide a valuable information on the electron-phonon-coupling strength and, second, no large-scale investigations of these properties by ab initio theoretical calculations appeared so far .

We calculate the electrical and thermal resistivity using the low-order variational approximation (LOVA) and our theoretical transport spectral functions found after Eq. (13). As follows from Eqs. (14) and (15) at high temperatures:

$$
\begin{gathered}
\rho=\frac{\pi \Omega_{c e l l} k_{B} T}{N\left(\epsilon_{F}\right)\left\langle v_{x}^{2}\right\rangle} \lambda_{t r}, \\
w=\frac{6 \Omega_{c e l l}}{\pi k_{B} N\left(\epsilon_{F}\right)\left\langle v_{x}^{2}\right\rangle} \lambda_{t r},
\end{gathered}
$$

and an important information is contained in the transport constant $\lambda_{t r}$ defined by

$$
\lambda_{t r}=2 \int_{0}^{\infty} \frac{d \omega}{\omega} \alpha_{t r}^{2} F(\omega) .
$$

It is usually believed that the latter is close to the superconducting $\lambda$ because the expressions for $\alpha_{t r}^{2} F(\omega)$ and $\alpha^{2} F(\omega)$ are quite similar, except for the factor $\left[1-\mathbf{v}(\mathbf{k}) \mathbf{v}\left(\mathbf{k}^{\prime}\right) /|\mathbf{v}(\mathbf{k})|^{2}\right]$ which preferentially weights the backscattering processes. However, there may exist a significant difference between $\alpha_{t r}^{2} F(\omega)$ and $\alpha^{2} F(\omega)$ for the case of strongly nested Fermi surfaces 56 due to the contribution from the backscattering of electrons between the opposite sides of the nested Fermi surface.

Despite the complexity of the Fermi surfaces in the transition metals we have obtained the transport functions $\alpha_{t r}^{2} F(\omega)$ quite close to the superconducting $\alpha^{2} F(\omega)$ which have been shown in Fig.1. The latter is also true for the simple metals considered in this work. Unfortunately, we have not investigated an interesting question 
about the low-frequency behavior of the $\alpha_{t r}^{2} F(\omega)$ due to a relatively coarse grid of the phonon wave vectors used for integrating the Eq. (13). The values of $\lambda_{t r}$ calculated from our transport functions are listed in Table IV. Comparison between $\lambda_{t r}$ and superconducting constants calculated earlier (Table II) gives the difference between them within $20 \%$ in all the tested materials. This is in agreement with previous conclusions that $\lambda_{t r} \sim \lambda$ for transition metals 57 .

The results of our calculated electrical resistivity $\rho(T)$ and thermal conductivity $w^{-1}(T)$ are presented, respectively, in Fig.3 and Fig.4 (full lines), up to the temperatures $500 K$. Symbols denote different measured points available from Refs. 58,59. (The residual values of the electrical resistivities are subtracted.) For the comparison with the experiment we are limited by the temperatures $T<2 \Theta_{t r}$, where $\Theta_{t r} \sim \sqrt{\left\langle\omega^{2}\right\rangle_{t r}}$ is close to the average phonon energy. (We list our calculated values of $\Theta_{t r}$ in Table IV.) This is so because the description of the transport properties at high temperatures require to take into account the unharmonicity effects and the Fermi-surface smearing. At low temperatures (usually when $\left.T<\Theta_{t r} / 5\right)$ the calculations will demand the inclusion of the $\mathrm{N}$-sheet and the inelasticity corrections beyond LOVA57. Also, here a more careful integration over the Brillouin zone is necessary to produce a correct limit of $\alpha_{t r}^{2} F(\omega)$ when $\omega \rightarrow 0$. Moreover, we cannot consider very low temperatures because of the effects of electronelectron scattering, size effects, impurity scattering, etc, which may give considerable contributions in addition to the electron-phonon scattering. The latter is basically responsible for the electrical resistivity of a metal at high temperatures in the absence of spin fluctuations. For the thermal conductivity, the lattice contribution to the heat current also exist and must be taken into account at the temperatures at least less than $100 \mathrm{~K}$. From Table IV we see that, except lead, the values of $\Theta_{t r}$ in all other materials are well above the low-temperature region and the comparison of our results with the measured ones must be relevant at the intermediate temperatures.

To compare the theoretical transport constants with the experiment, we fit the measured data 58 for $\rho(T)$ by polynomial series

$$
\rho(T)=\sum_{i=1}^{n} c_{i} T^{3-2 i}
$$

at the temperatures $\Theta_{t r} / 2<T<2 \Theta_{t r}$ with $n=2$. (The accuracy of the fit varies within $3 \%$ if $n$ is increased). The empirical values $\lambda_{t r}^{e x p}$ were then found using the extracted coefficient $c_{1}$ as follows

$$
\lambda_{t r}^{e x p}=\frac{c_{1} \omega_{p}^{2}}{8 \pi^{2} k_{B}}
$$

where $\omega_{p}$ is our calculated bare plasma frequency

$$
\omega_{p}^{2}=\frac{8 \pi N\left(\epsilon_{F}\right)\left\langle v_{x}^{2}\right\rangle}{\Omega_{c e l l}}
$$

The obtained $\lambda_{t r}^{e x p}$ are shown in Table IV where they can be compared with our calculated $\lambda_{t r}$. Note that similar numerical estimates for $\lambda_{t r}^{e x p}$ can also be made by analyzing thermal conductivities because the measured Lorentz number approaches to the Sommerfeld value at the temperatures $T \geq \Theta_{t r}$.

For $\mathrm{Al}$ [Fig.3(a)] we have found a good greement between the theoretical and the experimental 5 resistivities at the whole interval of the intermediate temperatures. The corresponding values of the transport constants are $\lambda_{t r}^{\text {calc }}=0.37$ and $\lambda_{t r}^{e x p}=0.39$. The reduction of the coupling constant $\left(\lambda_{\text {calc }}=0.44\right)$ to the transport one is less than $20 \%$. There is also an agreement between the theoretical curve and the experimental points 5 for the thermal conductivity [Fig.4(a)] above $150 \mathrm{~K}$. The theory, however, underestimates $w^{-1}(T)$ at the lower temperatures. An obvious explanation here is the neglection of the lattice contribution to the thermal current. In fact, as it can be seen from Fig.4, such underestimation at the low temperatures exist in all other materials considered in this work.

A comparison with the experiment is complicated for lead because of its low phonon energies $\left(\Theta_{t r} \sim 75 K\right)$ and the importance of the unharmonicity effects already at the low temperatures. The latter can possibly explain our discrepancy in the calculated electrical resistivity behavior shown in Fig.3(b). The same disagreement exist in our results for the thermal conductivity, Fig.4(b). Here, the discrepancy is minimal at the temperatures near $75 K$ and grows fastly as the temperature increases mainly because of the linear decay of the measured thermal conductivity. This obviously contradicts with the LOVA behavior of $w^{-1}(T)$ and is consistent with our assumption on the importance of the unharmonicity. Unlike in the other considered metals, the computed value 1.19 of the transport constant here is significantly smaller than the electron-phonon $\lambda=1.68$. This reduction could also point out the importance of the anisotropy in the electron-phonon scattering as well as the Fermi-surface effects which are not well reproduced by LOVA. In the absence of a calculation beyond LOVA it is difficult to determine the main source of errors.

Measured resistivity for $\mathrm{Nb}$ starts to saturate at high temperatures. In fact, this effect is evident [Fig.3(d)] at the temperatures above $2 \Theta_{t r} \sim 400 \mathrm{~K}$ and it does not appear at the intermediate interval where the behavior of the resistivity only slightly deviates from the LOVA prediction. Comparing the calculated $\lambda_{t r}=1.17$ and the empirical values $\lambda_{t r}^{e x p}=1.11$ gives the agreement about $5 \%$. Like in $N b$, there is a complete agreement between the theoretical and the experimental data for $V$ [Fig.3(c)] at the temperatures $\Theta_{t r} / 5<T<2 \Theta_{t r}$ (calculated $\left.\Theta_{t r} \sim 260 K\right)$. The theoretical $\lambda_{t r}=1.15$ coincides with the $\lambda_{t r}^{e x p}$ found empirically. The applicability of our method to the description of the transport properties for both $V$ and $N b$ is also supported by comparing the thermal-conductivity data, Fig.4(c),(d). 
Fig.3(e) and Fig.4(e) present the results of our calculations in $T a$. For $w^{-1}(T)$, an excellent correspondence of the theoretical prediction with observed behavior is obtained, but $\rho(T)$ is underestimated in our calculation within $10-12 \%$. As a consequence, the evaluated $\lambda_{t r}^{\text {exp }}=0.93$ slightly exceeds the value 0.83 of the theoretical transport constant. The discrepancy is, in principle, within our computational errors. Note however, that the experimental behavior 58 of $\rho(T)$ used in evaluating $\lambda_{t r}^{\text {exp }}$ may depend on the sample purity and should be verified by several measurements. The disagreement can also be assigned to the influence of high-temperature effects in the vicinity of the upper limit $\left(2 \Theta_{t r} \sim 340 K\right)$ of the intermediate interval .

The results for $M o$ are given in Fig.3(f) and Fig.4(f). To avoid the influence of high-temperature effects we have dropped out the measured resistivity points at the temperatures above $300 K$ (the calculated $\Theta_{t r} \sim 290 K$ ) . Fitting for $T<300 K$ gives the result for the empirical transport constant $\lambda_{t r}^{e x p}=0.40$ which is close to our prediction, $\lambda_{t r}^{c a l c}=0.35$. The agreement in the thermalconductivity data is satisfactory for the whole intermediate interval.

The slope of the resistivity in $C u$, Fig.3(g), is also obtained quite accurate as in the other materials. The value of $\lambda_{t r}$ is only slightly overestimated in the calculation. The discrepancy in the calculated thermal conductivity, Fig.4(g), is larger and consists about $20 \%$ at the room temperature. We cannot explain such disagreement by the renormalization due to the Coulomb correlations because, having been proportional to the ratio $\lambda_{t r} / \omega_{p}^{2}$, both $\rho(T)$ and $w^{-1}(T)$ must be insensitive to this effect in the first order. The underestimation of the theoretical $w^{-1}(T)$ can point out the largeness of the lattice contribution to the thermal conductivity at the temperatures above $100 K$. Unfortunately, there is a number of known difficulties to extract the latter values from the experiment 60 . From the low-temperature data analysis 60 one may conclude that the contribution to the thermal resistivity, $w_{e-h}^{p}$, from the process of a phonon decay by emissing electron-hole pairs is very small for $C u$ because of the apparently weak electron-phonon coupling $(\lambda \sim 0.12-0.14)$. This supports our explanation for the obtained discrepancy.

Finally, the predicted transport properties of $P d$ are presented in Fig.3(h) and Fig.4(h). Like $C u$, this metal has $4 d^{10}$ electronic configuration, but, in contrast to $C u$, we have found a very good agreement between the calculated curve $w^{-1}(T)$ and its measured behavior. We can consequently judge that the thermal conductivity carried by phonons is small in this case which is consistent with the conclusion 60 that the contribution $w_{e-h}^{p}$ is large for $P d$. We have also found an underestimation of the electrical resistivity in the calculation. The agreement between $\lambda_{t r}$ and $\lambda_{t r}^{\exp }$ (see Table IV) consists about 15\% which is, in principle, the upper limit of our computational uncertainty. Most likely, however, that the additional spin fluctuation mechanism of the resistivity is also present in this metal.

In summary, the behavior of $\rho(T)$ and $w(T)$ is consistent with the results (19), (20) at the intermediate temperatures and there is no significant discrepancy between our calculations and the experimental points. More precisely, we have extracted the values of $\lambda_{t r}^{\exp }$ using the experimental data for $\rho(T)$ together with our bandstructure value of $\omega_{p}$ and found the agreement between the experimental and the theoretical transport constants to be about $10 \%$ (in particular, lower than $5 \%$ for $A l, N b, M o$ and $V)$. In fact, compared with the experiment is the ratio $\lambda_{t r} / \omega_{p}^{2}$. Except possibly $C u$ and $P d$, the DFT-based band-structure calculations assumed to provide the proper magnitude for the plasma frequency. So, we drop the possibility of error cancellations and conclude that the theoretical $\lambda_{t r}$ are in the real agreement with the experiment. Relatively high error level in $\mathrm{Pb}$ can be explained by the importance of the unharmonic effects. The large lattice contribution to the thermal conductivity could affect our comparison for $C u$. In $P d$, the additional mechanism of the resistivity can also take place. Nevertheless, taking into account the agreement in the other calculated properties, we think that our description of the electronic transport in the considered materials is quite satisfactory.

\section{CONCLUSION}

We have presented $a b$ initio linear-response calculations of the electron-phonon interaction in the transition metals $C u, M o, N b, P d, T a, V$ and in the $s p$-metals $A l, P b$ using the local density functional method and the LMTO basis set. Our results for the lattice dynamical, superconducting and transport properties in these materials agree well with the experiment. They can be summarized as follows: (i) we have obtained tunneling spectral functions $\alpha^{2} F(\omega)$ and their first reciprocal moments $\lambda$ close to the measured ones; (ii) the correct values for the superconducting energy gap have been found using our calculated $\alpha^{2} F(\omega)$ and $\mu^{*}$ corresponding to the experimental $T_{c}$; (iii) the solution of the Eliashberg equation for $T_{c}$ (or for $\mu^{*}$ if $T_{c}$ is fixed) is well approximated by the conventional McMillan formula; (iv) the mass enhancement observed in the specific-heat measurements corresponds very well to our calculations and there is no paramagnon contribution in all the metals except $P d$; (v) we have found the electrical and thermal resistivities in agreement with the measured data; (vi) we have also found them to be well described by the LOVA expressions; (vii) the theoretical transport constants agree with the values of $\lambda_{t r}^{e x p}$ within $10 \%$. To summarize all these results, we conclude that our method gives the description of the electron-phonon coupling with the accuracy of order $10 \%$. We also conclude that the effect of renormalization of the energy bands due to electron-electron interactions is small in the considered materials. Some discrepancies between the theoretical and the tunneling 
values of $\lambda$ in $N b$ and $V$ can be assigned to the difficulty in processing the tunneling data. Nevertheless, it seems to us that more experimental and theoretical work is necessary to account for the large $\lambda$ and $\mu^{*}$ in $N b$ and, especially, in $V$.

\section{Acknowledgments.}

The authors are indebted to O. K. Andersen, E. G. Maksimov, O. V. Dolgov, O. Jepsen, A. Liechtenstein, I. I. Mazin, and S. Shulga for many helpful discussions. One of us (D. Y. S) was partially supported by INTAS(932154), ISF(MF-8300) and RFFI grants.

${ }^{1}$ For a review, see, e.g., G. Grimvall, in Electron-Phonon Interactions in Metals, edited by E. P. Wohlfarth (NorthHolland, Amsterdam, 1981).

${ }^{2}$ For a review, see, e.g., G. Gladstone, M. A. Jensen, and J. R. Schrieffer, in Superconductivity, edited by R. D. Parks (Marcel Dekker, New York, 1969), Vol.2.

${ }^{3}$ For a review, see, e.g., E. L. Wolf, in Principles of Electronic Tunneling Spectroscopy, (Oxford University Press, 1985).

${ }^{4}$ P. Hohenberg, W. Kohn, Phys. Rev. 136, B864 (1964).

${ }^{5}$ W. Kohn, L. J. Sham, Phys. Rev. 140, A1133 (1965).

${ }^{6}$ For a review, see, e.g., Theory of the Inhomogeneous Electron Gas, edited by S. Lundqvist and S. H. March (Plenum, New York, 1983).

${ }^{7}$ For a review, see, e.g., B. M. Klein and W. Pickett, in Superconductivity in $d$ - and $f$-Band Metals, edited by W. Buckel and W. Weber (Kernforschungszentrum, Karlsruhe, 1982), p. 477.

${ }^{8}$ L. J. Sham and J. M. Ziman, in Solid State Physics, edited by H. Ehrenreich and D. Turnbull (academic, New York, 1963), Vol. 15, p. 221.

${ }^{9}$ G. D. Gaspary and B. L. Gyorfy, Phys. Rev. Lett., 28, 801 (1972).

${ }^{10}$ W. H. Butler, in Physics of Transition Metals, (Inst. Phys. Conf. Ser. 55, 1980), p.505.

${ }^{11}$ For a review of supercell and perturbative approaches, see, e.g., Ab Initio Calculations of Phonon Spectra, edited by J. T. Devreese, V. E. Van Doren, and P. E. Van Camp (Plenum, New York, 1983).

12 M. M. Dacorogna, M. L. Cohen, and P. K. Lam, Phys. Rev. Lett. 55, 837 (1985).

13 T. W. Barbee, A. Garcia, M. L. Cohen, and J. L. Martins, Phys. Rev. Lett. 62, 1150 (1989); R. E. Cohen, W. H. Pickett, and H. Krakauer, Phys. Rev. Lett. 64, 2575 (1990).

14 A. I. Liechtenstein, I. I. Mazin, C. O. Rodrigues, O. Jepsen, O. K. Andersen, and M. Methfessel, Phys. Rev. B 44, 5388 (1991).

${ }^{15}$ H. Winter, J. Phys. F 11, 2283 (1981).
${ }^{16}$ S. Baroni, P. Gianozzi, and A. Testa, Phys. Rev. Lett. 58, 1861 (1987); N. E. Zein, Fiz. Tverd. Tela (Leningrad) 26, 3028 (1984) [Sov. Phys. Solid State 26, 1825 (1984)].

${ }^{17}$ R. M. Sternheimer, Phys. Rev. 96, 951 (1954); ibid. 107, 1565 (1957); ibid. 115, 1198 (1959).

18 See, e.g., P. Giannozzi, S. Gironcoli, P. Pavone and S. Baroni, Phys. Rev B 43, 7231 (1991); D. Kh. Blat, N. E. Zein and V. I. Zinenko, J. Phys. F 3, 5515 (1991); X. Gonze, D. C. Allan, and M. P. Teter, Phys. Rev. Lett. 68, 3603 (1992).

19 S. Y. Savrasov, Phys. Rev. Lett. 69, 2819 (1992).

${ }^{20}$ S. Y. Savrasov and E. G. Maksimov, Uspekhi Fiz. Nauk 165, 773 (1995), (English translation: Physics Uspekhi, in press).

${ }^{21}$ S. Y. Savrasov and O. K. Andersen, submitted to Phys. Rev. B.

22 O. K. Andersen, Phys. Rev. B 12, 3060 (1975).

${ }^{23}$ S. Y. Savrasov, D. Y. Savrasov, and O. K. Andersen, Phys. Rev. Lett. 72, 372 (1994).

${ }^{24}$ P. B. Allen, Phys. Rev. B 6, 2577 (1972).

${ }^{25}$ G. M. Eliashberg, Zh. Eksp. Teor. Fiz. 38, 966 (1960) (Sov. Phys. JETP 11, 696 (1960)).

${ }^{26}$ S. Y. Savrasov and D. Y. Savrasov, Phys. Rev. B 46, 12181 (1992).

27 P. B. Allen, Phys. Rev. B 31, 305 (1971).

${ }^{28}$ H. J. Monkhorst and J. D. Pack, Phys. Rev. B 13, 5188 (1976).

${ }^{29}$ P. Blöchl, O. Jepsen and O. K. Andersen, Phys. Rev. B 49, 16223 (1994).

${ }^{30}$ P. B. Allen, Phys. Status Solidi B 120, 529 (1983).

${ }^{31}$ U. von Barth and L. Hedin, J. Phys. C 5, 1629 (1972); V. L. Moruzzi, J. F. Janak, and A. R. Williams, Calculated Electronic Properties of Metals, (Pergamon, New York, 1978)

${ }^{32}$ D. M. Ceperley and B. J. Alder, Phys. Rev. Lett. 45, 566 (1980).

${ }^{33}$ S. H. Vosko, L. Wilk, and M. Nusair, Can. J. Phys. 58, 120 (1980)

34 Landolt-Börnstein, New Series, Group III, Vol. 13, subvol. a (Springer-Verlag, Berlin 1981).

35 S. Gironcoli, Phys. Rev. B 51, 6773 (1995).

${ }^{36}$ J. P. Perdew and Y. Wang, Phys. Rev. B 33, 8800 (1986).

${ }^{37}$ H. K. Leung, J. P. Carbotte, D. W. Taylor, and C. R. Leavens, Can. J. Phys. 54, 1585 (1976); see also Ref. 3.

38 See, e.g., W. L. McMillan and J. M. Rowell, in Superconductivity, edited by R. D. Parks (Marcel Dekker, New York, 1969), Vol.1.

${ }^{39}$ J. Bostock, V. Diadiuk, W. N. Cheung, K. H. Lo, R. M. Rose, and M. L. A. MacVicar, Phys. Rev. Lett. 36, 603 (1976); K. Gärtner and A. Hahn, Z. Naturforsch. 31a, 361 (1976).

${ }^{40}$ B. Robinson, T. H. Geballe and J. M. Rowell, in Superconductivity in d-and f-band metals, AIP Conf. 1972, p.381

${ }^{41}$ G. B. Arnold, J. Zasadzinski and E. L. Wolf, Phys. Lett. 69A, 136 (1978); E. L. Wolf, J. Zasadzinski, J. W. Osmun and G. B. Arnold, J. Low Temp. Phys. 40, 19 (1980);

${ }^{42}$ M. J. Bostock, M. L. A. MacVicar, G. B. Arnold, J. Zasadzinski and E. L. Wolf, in Proceedings of the Third International Conference on Superconductivity of $d$ - and $f$-band metals, ed. by H.Suhl and M.B.Maple (Academy Press, New York 1980), p.153. 
${ }^{43}$ D. A. Papaconstantopoulus, L. L. Boyer, B. M. Klein, A. R. Williams, V. L. Moruzzi, and J. F. Janak, Phys. Rev. B 15, 4221 (1977).

${ }^{44}$ W. H. Butler, H. G. Smith, and N. Wakabayashi, Phys. Rev. Lett. 39, 1004, (1977); W. H. Butler, F. J. Pinski, and P. B. Allen, Phys. Rev. B 19, 3708 (1979).

${ }^{45}$ B. N. Harmon and S. K. Sinha, Phys. Rev. B 16, 3919 (1977); D. Glötzel, D. Rainer, and H. R. Schober, Z. Phys. B 35, 317 (1979); M. Peter, T. Jarlborg, M. Dacorogna, and E. Moser, in Superconductivity in $d$ - and f-Band Metals, 1982 (Ref. 月), p.515.

${ }^{46}$ F. J. Pinski and W. H. Butler, Phys. Rev. B. 19, 6010 (1979).

47 W. L. McMillan, Phys. Rev. 167, 331 (1968).

${ }^{48}$ P. B. Allen and R. C. Dynes, Phys. Rev. B 12, 905 (1975).

49 J. Zasadzinski, D. M. Burnell, E. L.Wolf and G. B. Arnold, Phys. Rev. B 25, 1622 (1982).

${ }^{50}$ L. Y. Shen, Phys. Rev. Lett. 24, 1104 (1970).

${ }^{51}$ G. W. Crabtree, D. H. Dye, D. P. Karim, D. D. Koelling, and J. B. Ketterson, Phys. Rev. Lett. 42, 390 (1979).

${ }^{52}$ See Ref. 3, p. 263, and references therein.

${ }^{53}$ H. Rietschel and H. Winter, Phys. Rev. Lett. 43, 1256 (1979).

${ }^{54}$ K. A. Geschneidner, in Solid State Physics, ed. by H. Ehrenreich, F. Seitz and D. Turnbull (Academic Press, New York, 1964), vol.16, p. 275.

${ }^{55}$ R. Radebaugh and P. H. Keesom, Phys. Rev. 149, 217 (1966)

${ }^{56}$ V. H. Grespi and M. L. Cohen, Solid State Commun. 81, 187 (1992).

${ }^{57}$ E. J. Pinski, P. B. Allen, W. H. Butler, Phys. Rev. B 23, 5080 (1981).

${ }^{58}$ Landolt-Börnstein, New Series, Group III, Vol. 15, subvol. a (Springer-Verlag, Berlin, 1982).

${ }^{59}$ Landolt-Börnstein, New Series, Group III, Vol. 15, subvol. c (Springer-Verlag, Berlin, 1982).

${ }^{60}$ W. H. Butler and R. K. Williams, Phys. Rev. B 18, 6483 (1978).

TABLE I. Comparison between calculated and experimental 34 phonon frequencies $(\mathrm{THz})$ at the high-symmetry points $X, L$ for the fcc metals $A l, P b, C u, P d$, and at the points $H, N$ for the bcc metals $V, N b, T a, M o$. Also listed are the theoretical-to-experimental volume ratios $V / V_{0}$ used in the calculations.

\begin{tabular}{|c|c|c|c|c|c|c|c|c|c|}
\hline$\overline{f c c} \| \mathrm{bcc}$ & & $A l$ & $\mathrm{~Pb}$ & $V$ & $\mathrm{Nb}$ & $T a$ & $M o$ & $C u$ & $P d$ \\
\hline \multirow{2}{*}{$X_{L} \| H_{L T}$} & theory & 9.51 & 1.80 & 8.03 & 6.43 & 5.13 & 5.71 & 7.69 & 7.17 \\
\hline & exp. & 9.69 & 1.86 & - & 6.49 & 5.03 & 5.52 & 7.25 & 6.72 \\
\hline \multirow{2}{*}{$X_{T} \| N_{L}$} & theory & 5.83 & 1.06 & 7.22 & 5.52 & 4.54 & 7.99 & 5.36 & 5.01 \\
\hline & exp. & 5.78 & 0.89 & - & 5.66 & 4.35 & 8.14 & 5.13 & 4.64 \\
\hline \multirow{2}{*}{$L_{L} \| N_{T_{1}}$} & theory & 9.84 & 2.18 & 4.76 & 3.94 & 2.65 & 5.74 & 7.77 & 7.39 \\
\hline & exp. & 9.69 & 2.18 & - & 3.93 & 2.63 & 5.73 & 7.30 & 7.02 \\
\hline \multirow{2}{*}{$L_{T} \| N_{T_{2}}$} & theory & 4.33 & 0.92 & 6.17 & 4.80 & 4.18 & 4.69 & 3.64 & 3.60 \\
\hline & exp. & 4.19 & 0.89 & - & 5.07 & 4.35 & 4.56 & 3.42 & 3.34 \\
\hline$V / V_{0}$ & & 0.955 & 1.002 & 0.990 & 0.972 & 0.974 & 0.971 & 0.985 & 0.975 \\
\hline
\end{tabular}


TABLE II. Comparison between the calculated electron-phonon coupling constants $\lambda_{\text {calc }}$ and the values of $\lambda_{\text {tun }}$ deduced from the tunnelling experiments. Also listed are the values of $\lambda_{s-h}$ extracted from the measured specific-heat coefficient $\gamma$ with the use of our calculated density of states $N\left(\epsilon_{F}\right)$.

\begin{tabular}{|c|c|c|c|c|c|c|c|c|}
\hline & $A l$ & $\mathrm{~Pb}$ & $V$ & $\mathrm{Nb}$ & $T a$ & $M o$ & $\mathrm{Cu}$ & $P d$ \\
\hline$\overline{\lambda_{\text {calc }}}$ & 0.44 & 1.68 & 1.19 & 1.26 & 0.86 & 0.42 & 0.14 & 0.35 \\
\hline$\lambda_{t u n}$ & $0.42^{a}$ & $1.55^{a}$ & $0.82^{a}$ & $1.04^{a}, 1.22^{b}$ & $0.78^{a}$ & - & - & - \\
\hline$\lambda_{s-h}$ & 0.43 & 1.64 & $1.00,1.17$ & 1.17 & 0.83 & 0.45 & - & 0.69 \\
\hline$N\left(\epsilon_{F}\right), \frac{\text { states }}{\text { Ry*cell }}$ & 5.49 & 6.87 & 26.14 & 20.42 & 18.38 & 8.34 & 4.36 & 34.14 \\
\hline$\gamma, \frac{m J}{K^{2} m o l}$ & $1.36^{c}$ & $3.14^{c}$ & $9.04^{c}, 9.82^{d}$ & $7.66^{c}$ & $5.84^{c}$ & $2.10^{c}$ & $0.69^{c}$ & $10.0^{c}$ \\
\hline
\end{tabular}

${ }^{a}$ Reference 3

${ }^{b}$ Reference 42

${ }^{c}$ Reference 54

${ }^{d}$ Reference 55

TABLE III. Calculated values of the Coulomb pseudopotential $\mu^{*}$ which provide the experimental values of $T_{c}$ as the solutions of the Eliashberg equation with our knowledge of $\alpha^{2} F(\omega)$. Values $T_{c}^{M c M}$ were then found with our $\omega_{l o g}$, $\lambda$ and $\mu^{*}$ in order to check the accuracy of the McMillan $T_{c}$-expression. Also shown are the computed and the measured 3 superconducting energy-gap parameters $\Delta_{0}$.

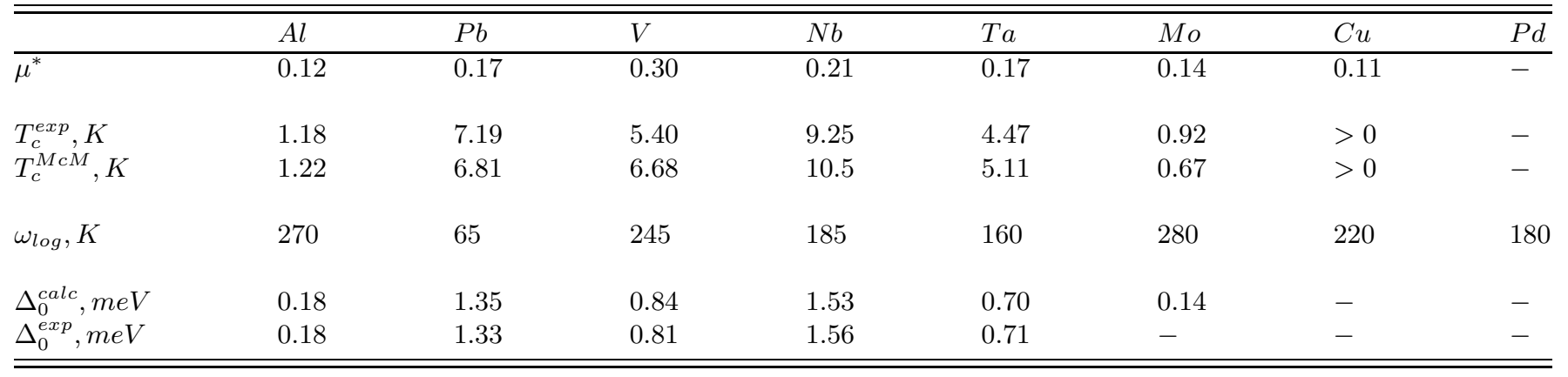

TABLE IV. Comparison between calculated and empirical values of the transport constant $\lambda_{t r}$. The values of $\lambda_{t r}^{e x p}$ were deduced from the electrical-resistivity data $\$ 2$ with help of our calculated bare plasma frequencies $\omega_{p}$. Also listed are the average transport frequencies $\Theta_{t r}=\sqrt{\left\langle\omega^{2}\right\rangle_{t r}}$

\begin{tabular}{llllllll}
\hline \hline & $A l$ & $P b$ & $V$ & $N b$ & $T a$ & $M o$ & $C u$ \\
\hline$\lambda_{t r}^{\text {calc }}$ & 0.37 & 1.19 & 1.15 & 1.17 & 0.83 & 0.35 & 0.13 \\
$\lambda_{t r}^{\text {exp }}$ & 0.39 & 1.52 & 1.15 & 1.11 & 0.93 & 0.40 & 0.12 \\
$\omega_{p}, e V$ & 12.29 & 14.93 & 7.95 & 9.47 & 9.05 & 8.81 & 0.43 \\
$\Theta_{t r}, K$ & 330 & 75 & 260 & 200 & 170 & 290 & 7.75 \\
\hline \hline
\end{tabular}


Fig.1(a)-(h). Calculated phonon-dispersion curves along several symmetry directions for the eight elemental metals considered in this work. The lines result from the interpolation between the theoretical points (circles). The results of avaiable neutron-difraction measurements 34 are shown by triangles. Also plotted are the calculated densities of states (DOS).

Fig.2(a)-(h). Calculated spectral functions $\alpha^{2} F(\omega)$ of the electron-phonon interaction (full lines) for the eight elemental metals considered in this work. The behavior of the electron-phenon prefactor $\alpha^{2}(\omega)$ is shown by dashed lines. Symbol plots present the results of available tunnelling experiments 33 .

Fig.3(a)-(h). Calculated temperature dependence of the electrical resistivity, $\rho(T)$, as a lowest-order variational solution of the Boltzmann equation for the eight elemental metals considered in this work. Symbols show different experimental data available from Ref. 58 .

Fig. 4(a)-(h). Calculated temperature dependence of the thermal conductivity, $w^{-1}(T)$, as a lowest-order variational solution of the Boltzmann equation for the eight elemental metals considered in this work. Symbols show different experimental data available from Ref. 59. 


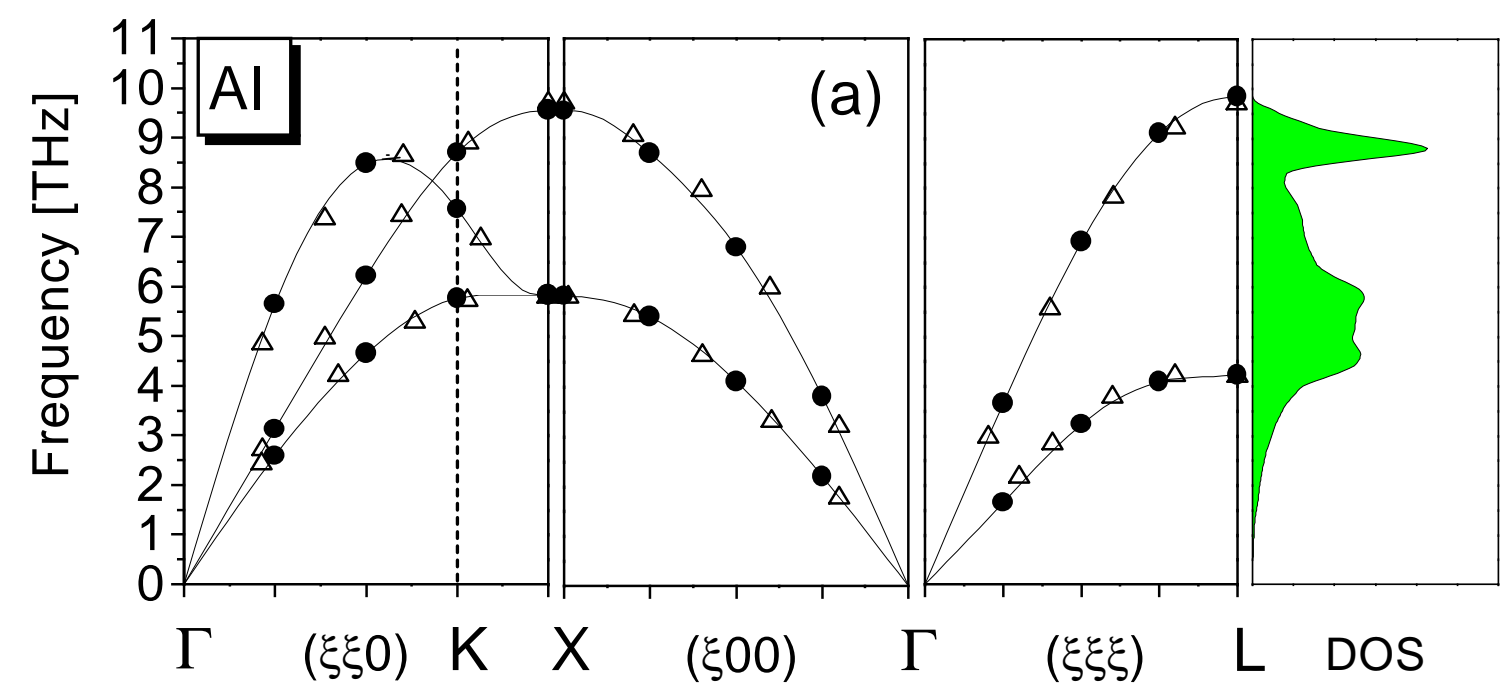



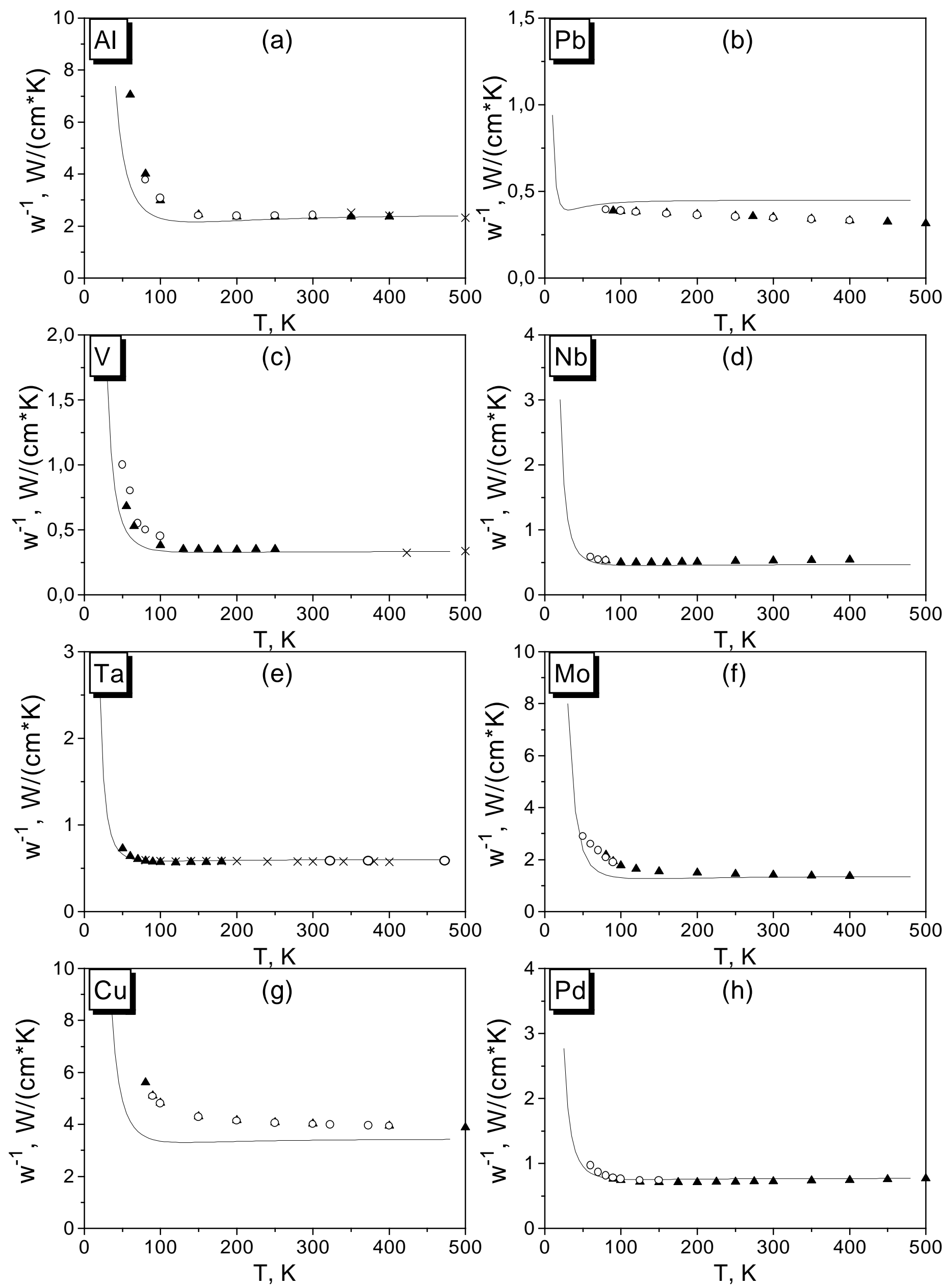


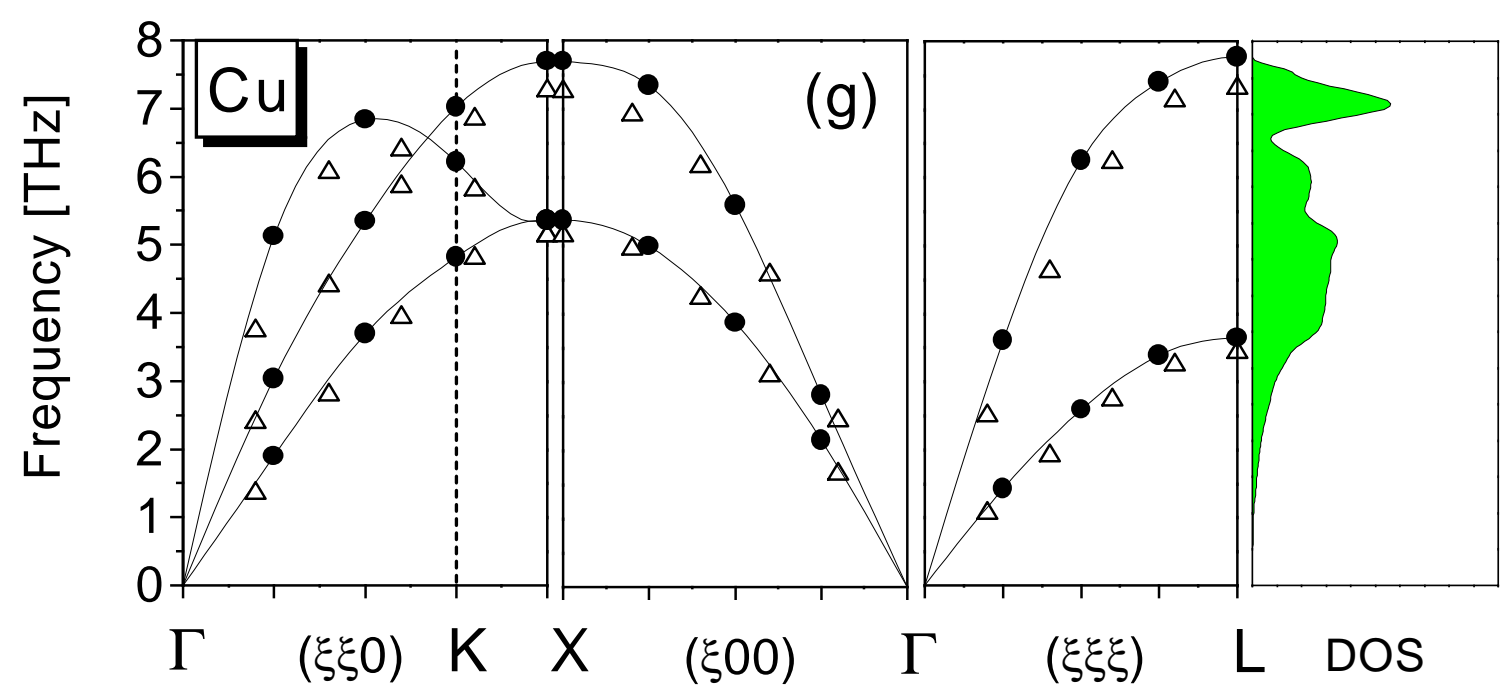



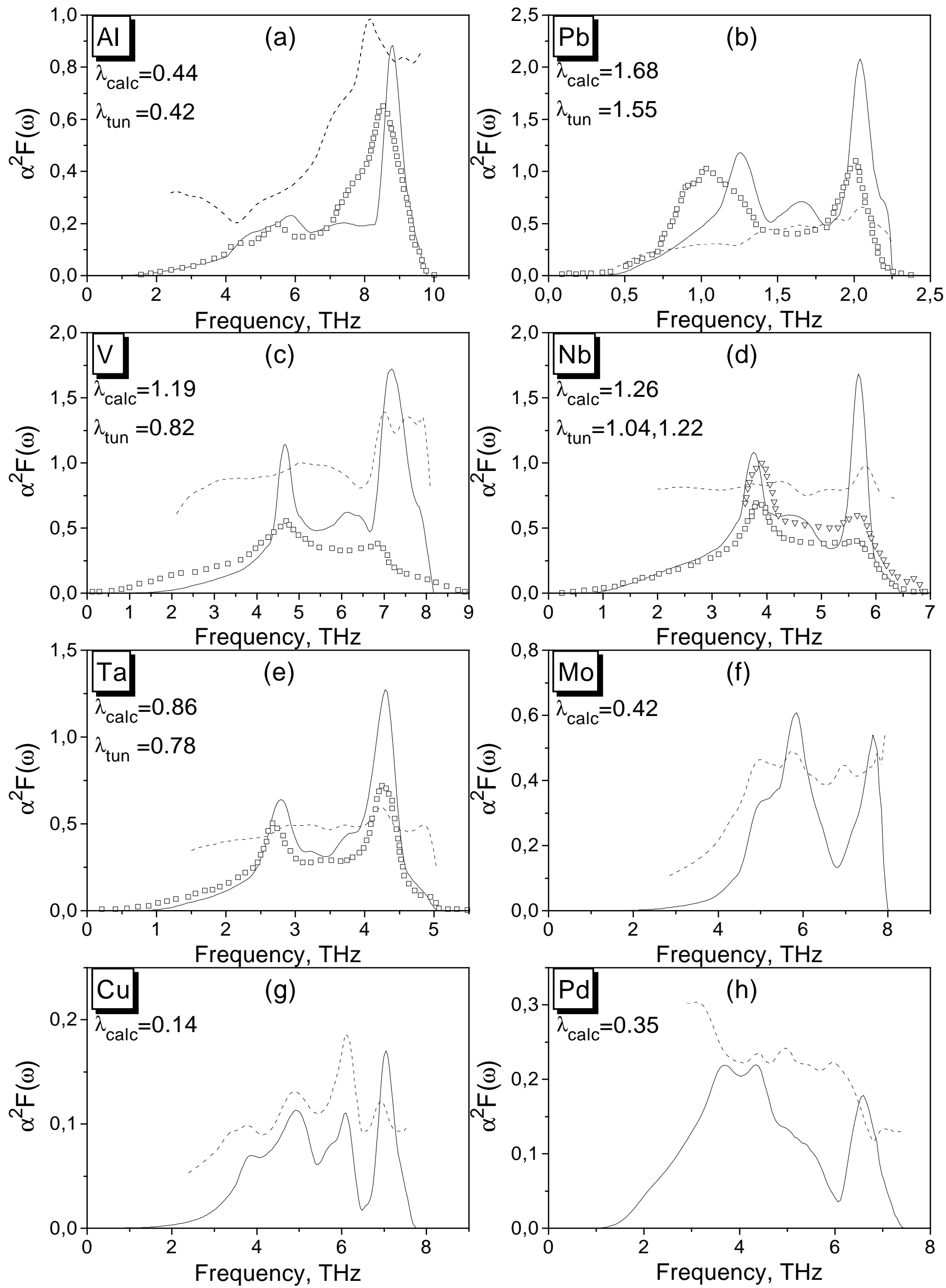


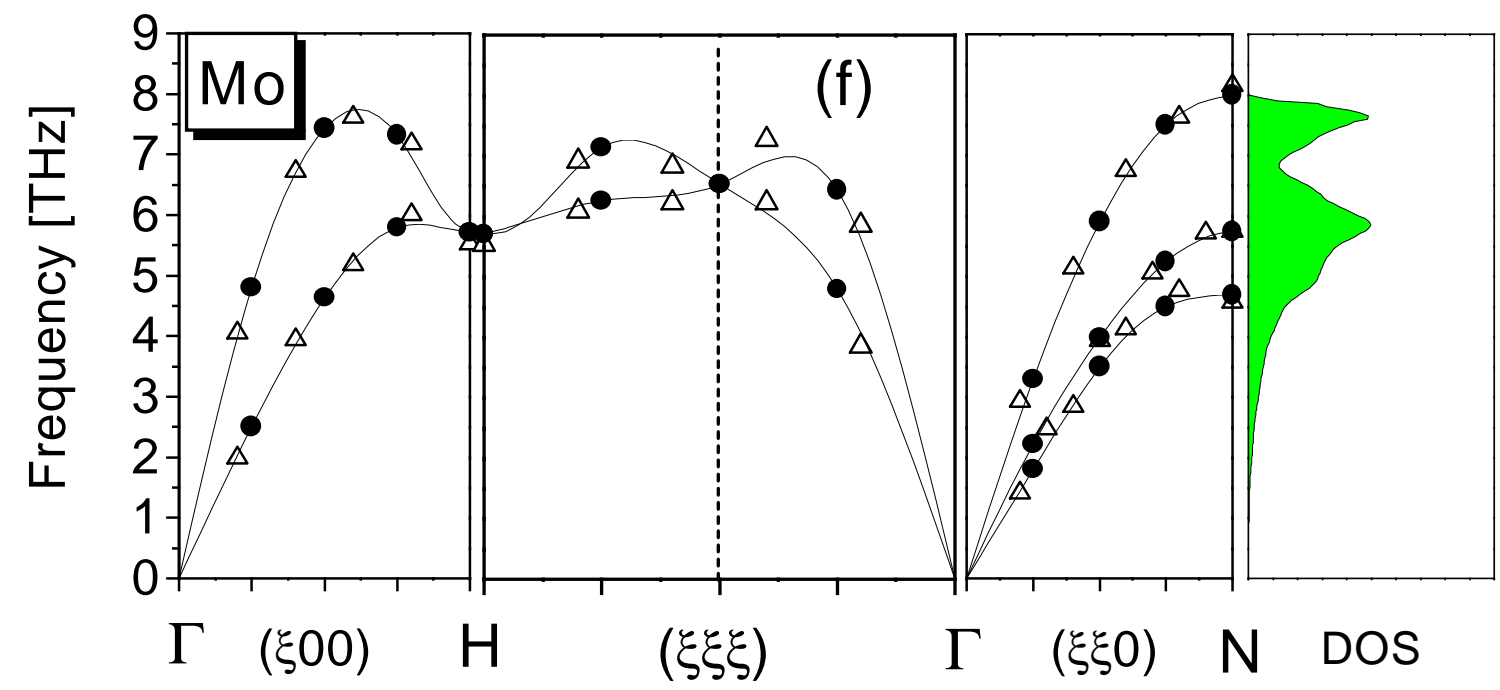




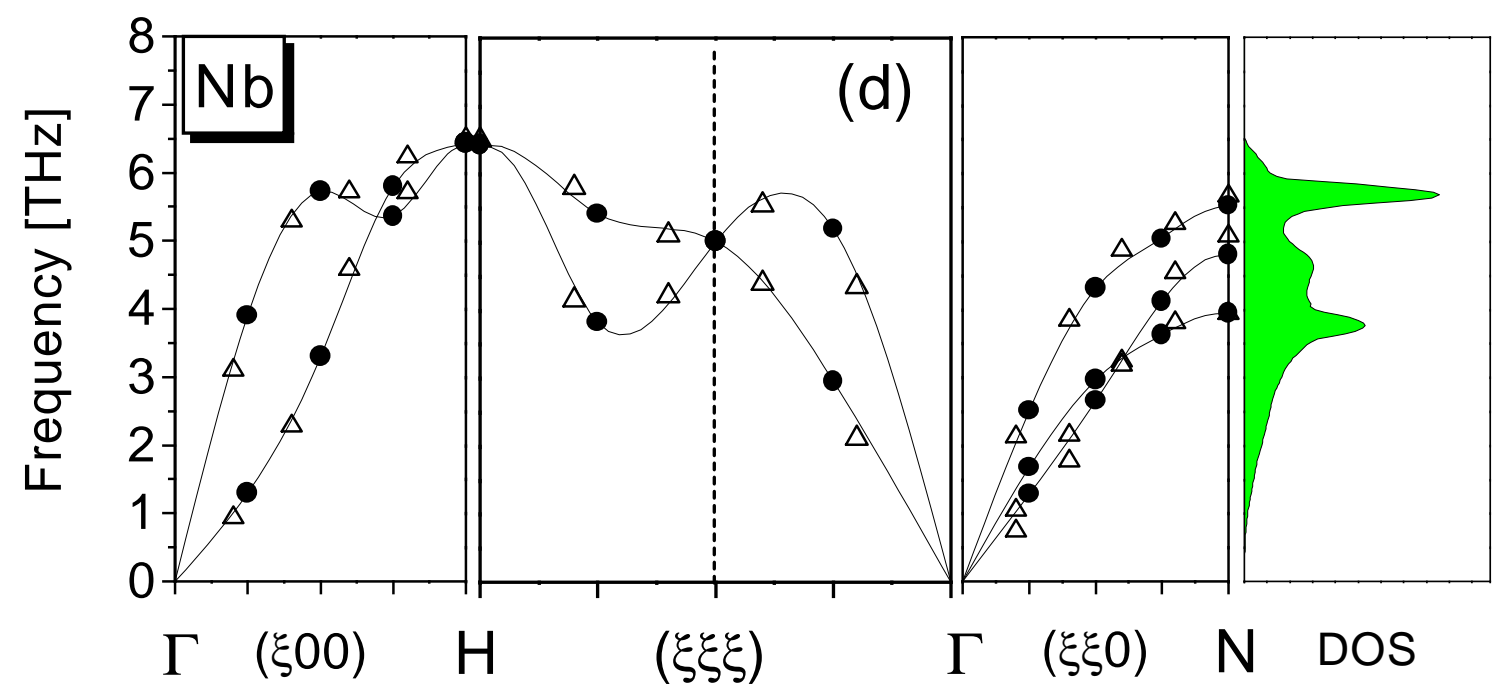




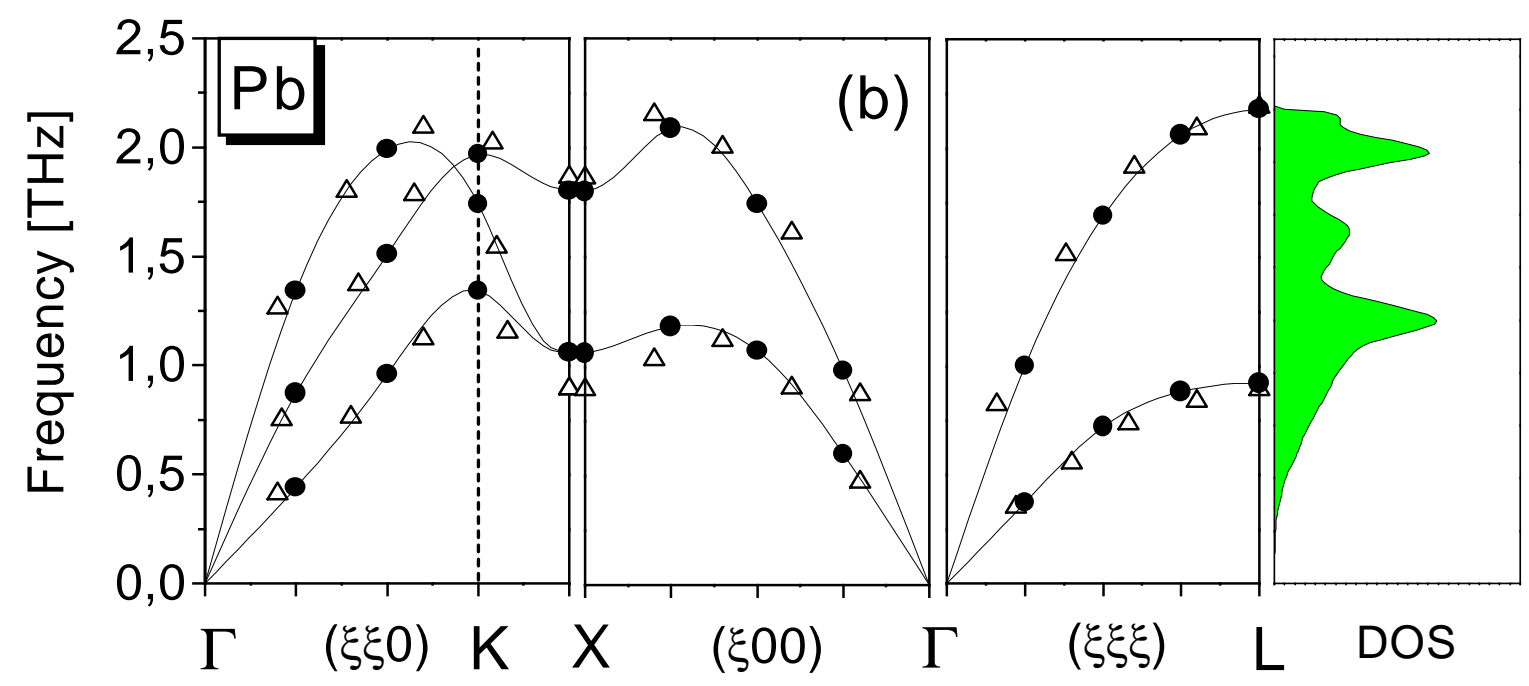




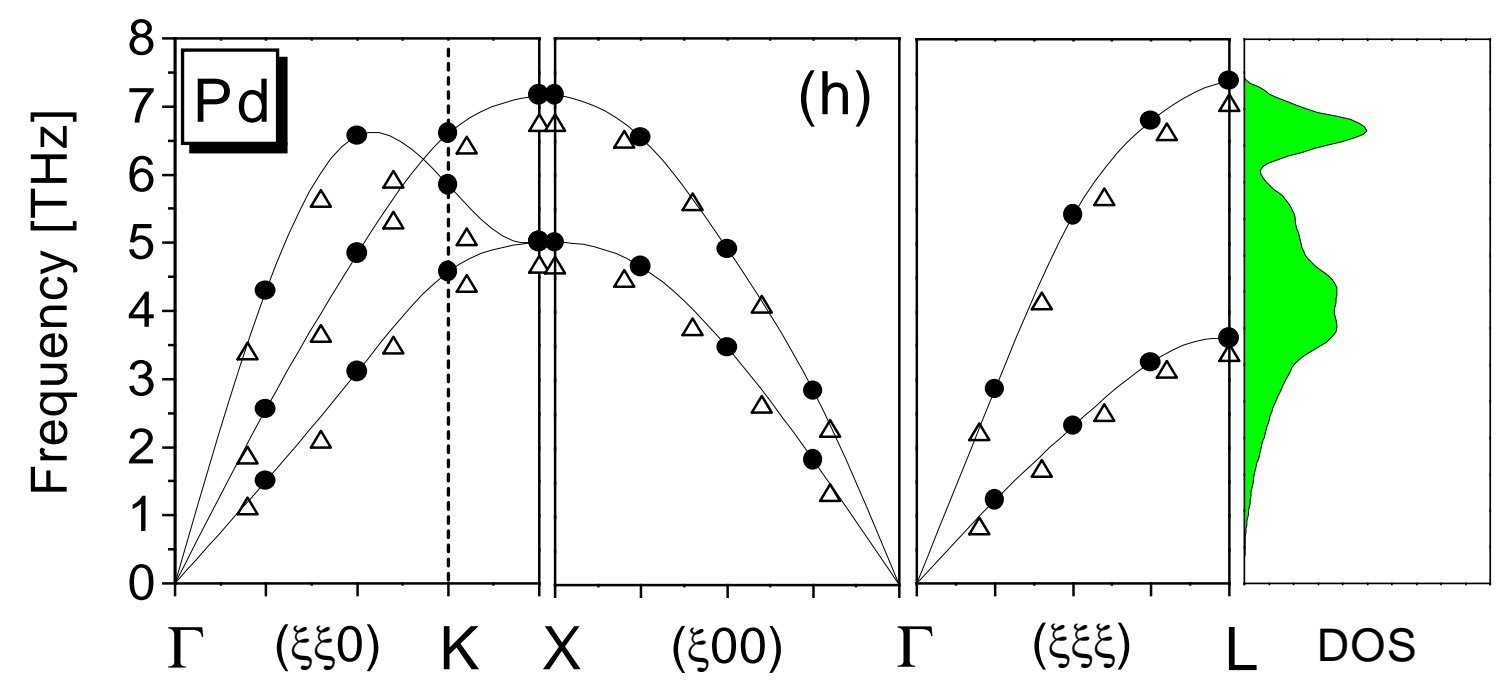



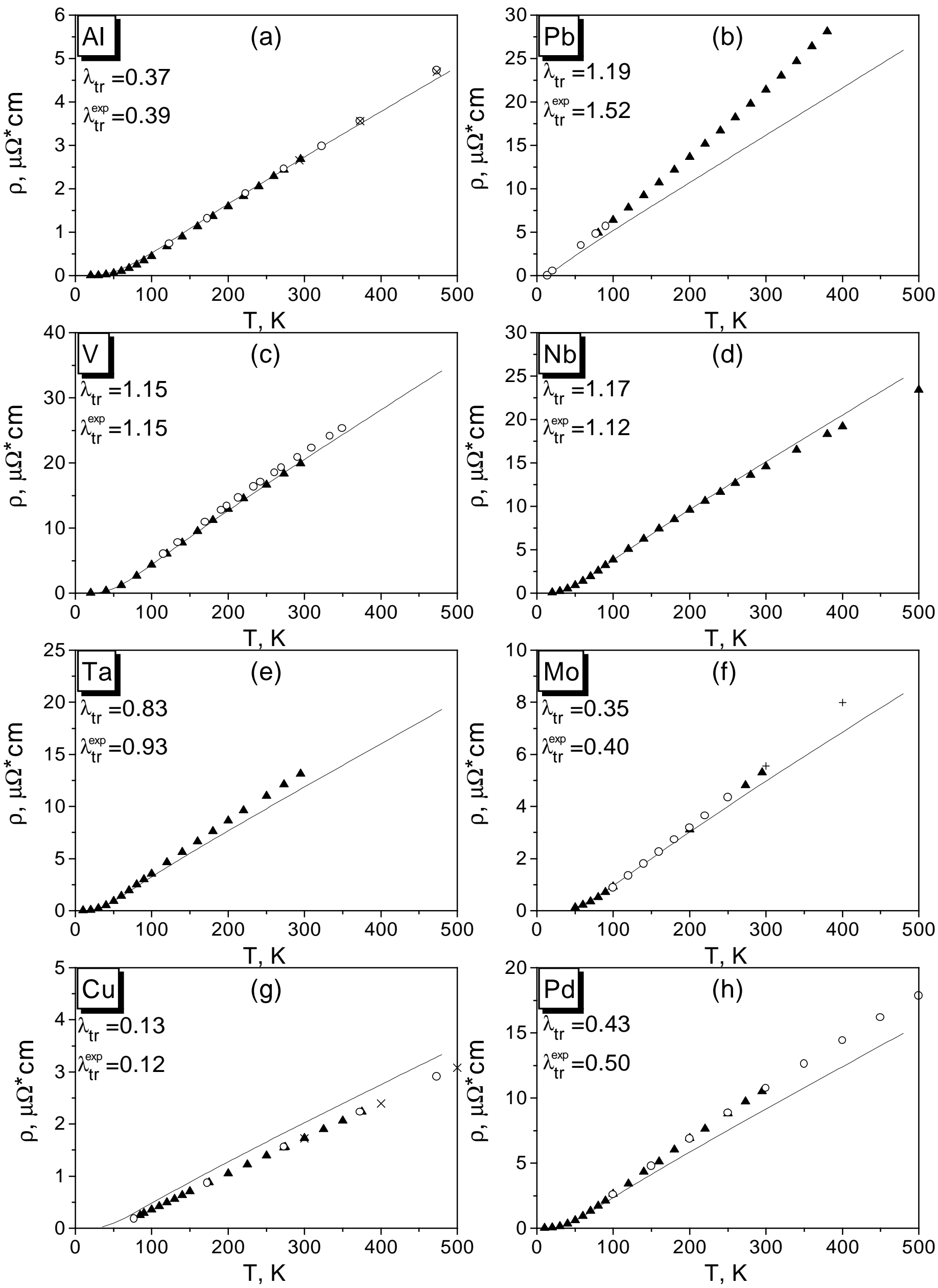


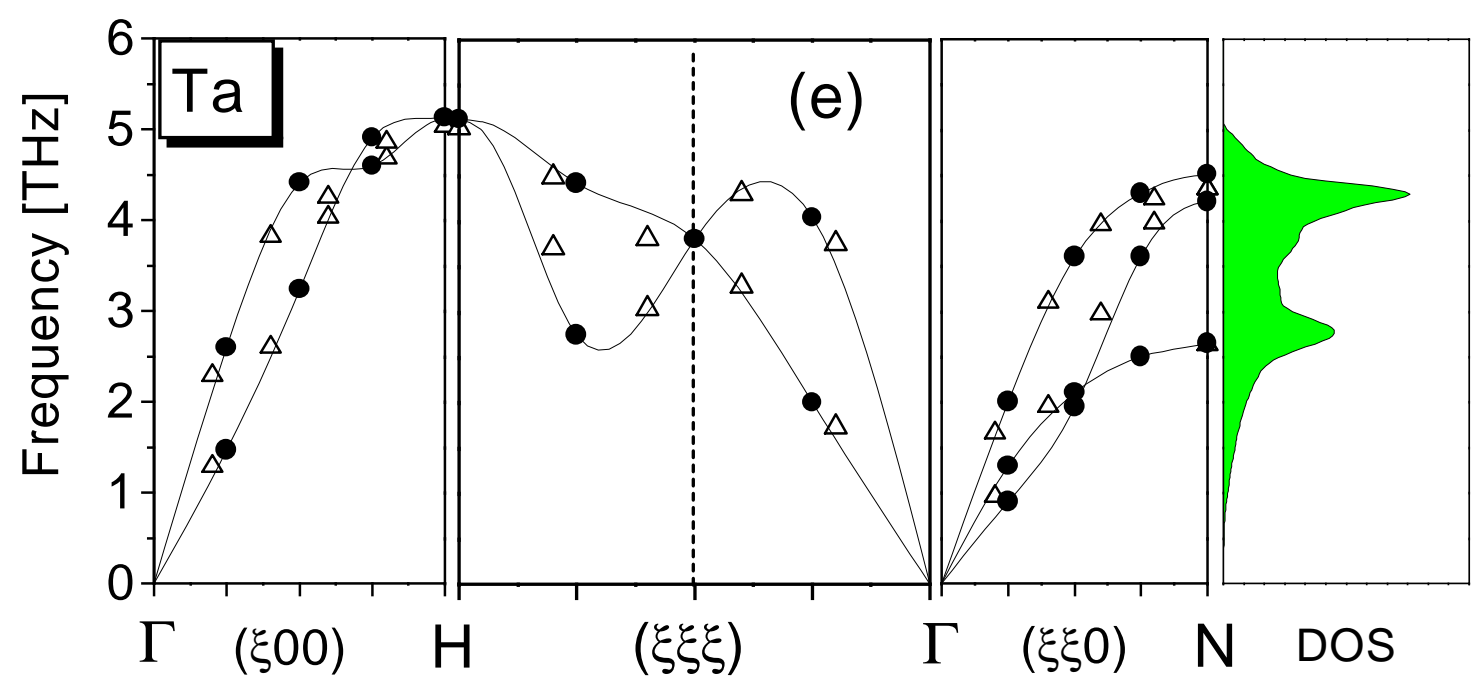




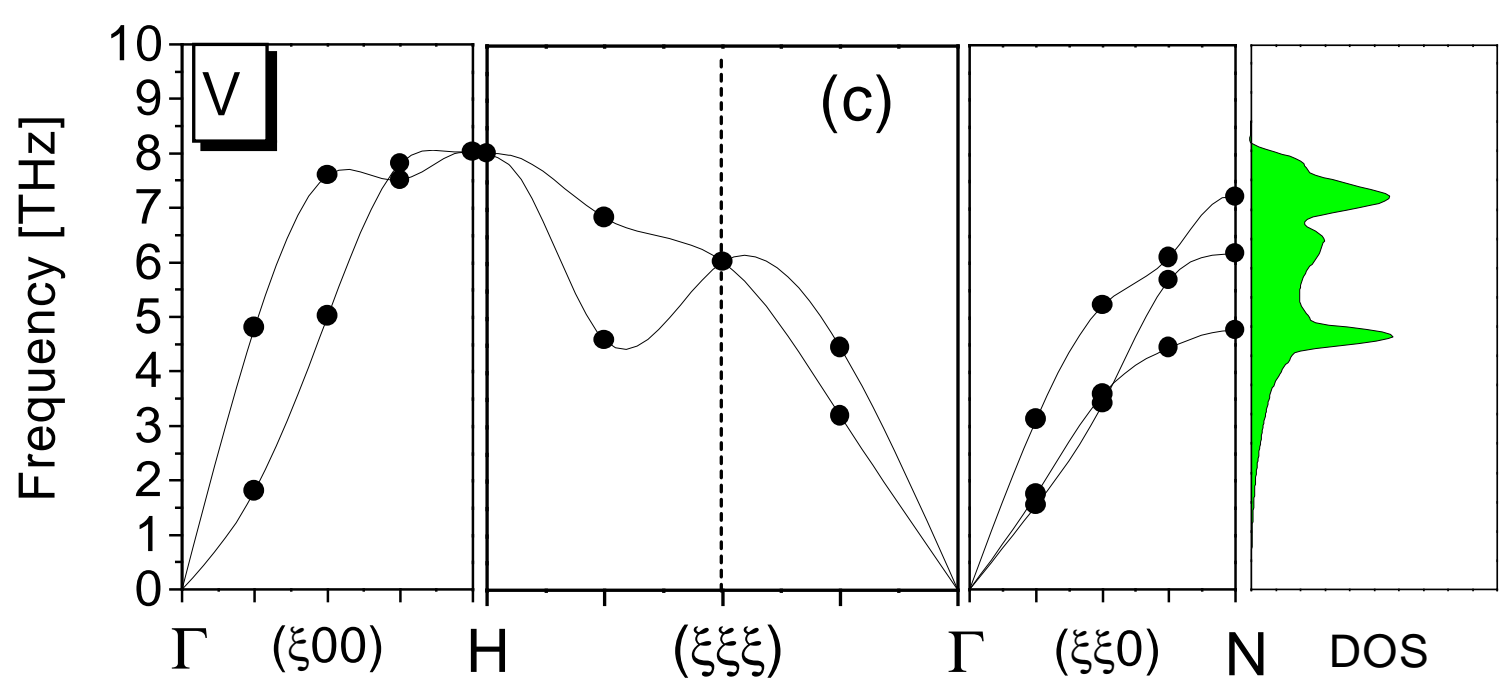

